\title{
THE COEVALITY OF YOUNG BINARY SYSTEMS
}

\author{
Adam L. Kraus and Lynne A. Hillenbrand \\ California Institute of Technology, Department of Astrophysics, MC 105-24, Pasadena, CA 91125, USA; alk@astro.caltech.edu \\ Received 2009 February 11; accepted 2009 August 28; published 2009 September 23
}

\begin{abstract}
Multiple star systems are commonly assumed to form coevally; they thus provide the anchor for most calibrations of stellar evolutionary models. In this paper, we study the binary population of the Taurus-Auriga association, using the component positions in an HR diagram in order to quantify the frequency and degree of coevality in young binary systems. After identifying and rejecting the systems that are known to be affected by systematic errors (due to further multiplicity or obscuration by circumstellar material), we find that the relative binary ages, $|\Delta \log \tau|$, have an overall dispersion $\sigma_{|\Delta \log \tau|} \sim 0.40$ dex. Random pairs of Taurus members are coeval only to within $\sigma_{|\Delta \log \tau|} \sim 0.58 \mathrm{dex}$, indicating that Taurus binaries are indeed more coeval than the association as a whole. However, the distribution of $|\Delta \log \tau|$ suggests two populations, with $\sim 2 / 3$ of the sample appearing coeval to within the errors $\left(\sigma_{|\Delta \log \tau|} \sim 0.16 \mathrm{dex}\right)$ and the other $\sim 1 / 3$ distributed in an extended tail reaching $|\Delta \log \tau| \sim 0.4-0.9$ dex. To explain the finding of a multipeaked distribution, we suggest that the tail of the differential age distribution includes unrecognized hierarchical multiples, stars seen in scattered light, or stars with disk contamination; additional follow-up is required to rule out or correct for these explanations. The relative coevality of binary systems does not depend significantly on the system mass, mass ratio, or separation. Indeed, any pair of Taurus members wider than $\sim 10^{\prime}(\sim 0.7 \mathrm{pc})$ shows the full age spread of the association.
\end{abstract}

Key words: binaries: general - Hertzsprung-Russell (HR) diagram - open clusters and associations: individual (Taurus-Auriga) - stars: evolution - stars: fundamental parameters - stars: pre-main sequence

Online-only material: color figures

\section{INTRODUCTION}

Stellar evolutionary models are critical for interpreting astronomical observations, but they are not well calibrated for pre-main sequence (PMS) stars. Such calibration requires the measurement of some or all of the fundamental stellar properties: age, mass, radius, luminosity, and effective temperature. Ages are notoriously difficult to estimate (Mamajek \& Hillenbrand 2008; Hillenbrand 2009), though they can be inferred indirectly from a membership in a stellar population for which the mean age can be determined. Stellar masses and/or radii require orbital monitoring of eclipsing or visual binary systems. The known pre-main sequence eclipsing binary systems sparsely sample parameter space due to their extreme rarity (e.g., Irwin et al. 2007; Stassun et al. 2007; Stempels et al. 2008). Most PMS visual binaries have only partial orbits because young stars are distant and any systems that can be spatially resolved necessarily have wide separations and corresponding long periods (Steffen et al. 2001; Duchêne et al. 2006), though a handful of short-period systems are bright enough for interferometric techniques to be feasible (Boden et al. 2005; Schaefer et al. 2008). In contrast to ages, masses, and radii, the luminosities and temperatures of stars are straightforward to infer from single-epoch observations, so they offer the best near-term prospects for the systematic calibration of stellar models. The procedure can also be inverted: given a star's luminosity and temperature, a theoretical model can be used to estimate its age and mass, plus its radius can be estimated directly from the Stefan-Boltzmann law.

The standard procedure for calibrating models with luminosities and temperatures is to place two or more nominally coeval stars on an HR diagram. These stars should trace an empirical isochrone sequence, and this sequence can be compared with theoretical isochrones in order to test their consistency with observations. The HR diagram analysis has provided many crucial insights into models of stellar interiors, atmospheres, and evolution (e.g., White et al. 1999; Luhman et al. 2003). There are many systematic astrophysical effects that can complicate this analysis, including unresolved multiplicity, obscuration from circumstellar material (i.e., an envelope or edge-on disk), and veiling from accretion (at blue optical wavelengths) or circumstellar disk emission (at near-infrared wavelengths). These effects can yield ages with errors of an order of magnitude or more, so the samples used in this analysis must be inspected closely to identify stars that might be affected. Additional physics, such as stellar activity or convection efficiency, could also play a role (Chabrier et al. 2007; Stassun et al. 2008).

The inverse procedure plays an important role in the study of binary systems (e.g., Hartigan et al. 1994; White \& Ghez 2001; Hartigan \& Kenyon 2003). It is commonly assumed that multiple star formation proceeds almost simultaneously, such that all stars in a bound multiple system are coeval. However, this assumption can be tested only by using the evolutionary models that require calibration, so any apparent disagreement between binary component ages could be due to noncoevality or errors in the models. Emerging evidence from several young eclipsing binary systems shows that their component properties are inconsistent with any single age predicted by pre-main sequence evolutionary models (e.g., Stassun et al. 2007, 2008), but the frequency and degree of implied noncoevality are still unclear.

In this paper, we estimate the ages of a large sample of stringently vetted young binary systems in the Taurus association ( $\tau \sim 1-2 \mathrm{Myr} ; d \sim 145 \mathrm{pc}$ ) in order to test the system components' relative coevality and the validity of theoretical isochrones in matching empirical HR diagram sequences. In Section 2, we describe our sample of binary systems, and in Section 3, we describe the stellar models and 
analysis techniques used to infer stellar ages. In Section 4, we show an HR diagram with all our sample members and identify likely contaminants. In Section 5, we test the coevality of young binary systems by adopting model-predicted ages. Finally, in two appendices, we test the evolutionary models using a large sample of likely single stars and using a subset of high-order multiple systems.

\section{THE SAMPLE}

The accurate determination of stellar parameters requires spatially and/or spectrally resolved observations that are not polluted by light from companions; our sample is comprised of all known Taurus binary systems that have spectral types and flux ratios for at least two components. At the distance of Taurus $(\sim 145 \mathrm{pc})$, most binary systems with separations of $\lesssim 100$ 200 AU cannot be resolved in seeing-limited observations with typical resolutions of $\sim 1^{\prime \prime}$. Given this limit, most sample members have been observed with Hubble Space Telescope (HST), ground-based adaptive optics, or echelle spectrographs (distinguishing the components' spectra and relative fluxes). High-order multiplicity can give the appearance of noncoevality, so we immediately omitted any binary pairs that contained an additional component without its own measured spectral type and flux. As we describe in Section 4, we also cut additional targets from this sample if they appeared to be affected by other systematic uncertainties. We list the observed properties of our sample members in Table 1, along with the references used to infer spectral types, extinctions, and fluxes. Given the ad hoc nature of our sample, it is not complete and may be subject to biases, but the goal of our initial sample selection was to be as inclusive as possible.

The wide components that could be resolved in seeinglimited observations were drawn from the sample studied in our previous wide multiplicity survey (Kraus \& Hillenbrand 2007a, 2009). Most of these stars had already been identified as Taurus members, so we drew their observed properties from previous work by Kenyon \& Hartmann (1995), Duchêne et al. (1999), White \& Basri (2003), White \& Hillenbrand (2004), and Luhman $(2004,2006)$. All of these authors reported spectral types and extinctions, and we adopted NIR magnitudes from the Two Micron All Sky Survey (2MASS) Point Source Catalog (Skrutskie et al. 2006) or from our own point-spread function (PSF) fitting photometry of 2MASS atlas images (Kraus \& Hillenbrand 2007a). The 2MASS photometry for RW Aur AB and most of the Duchêne et al. sample was unreliable since the system separations fell near the 2MASS resolution limits, so we adopted total $K$ fluxes from 2MASS and the $K$ band flux ratios reported by White \& Ghez (2001) or Correia et al. (2006). We adopted an outer separation limit of $30^{\prime \prime}$ for identifying binary pairs. The binary population probably dominates among all pairs extending out to $\sim 2^{\prime}$ (Kraus \& Hillenbrand 2008), but the frequency of chance alignments between unbound stars becomes significant at $>30^{\prime \prime}$.

The closer systems that could be resolved with highresolution imaging were drawn from several recent spectroscopic surveys. Most of the spectroscopic observations were obtained with HST/Space Telescope Imaging Spectrograph (STIS) by Hartigan \& Kenyon (2003), but several systems were observed under good seeing by Duchêne et al. (1999). Individual systems were also studied by White et al. (1999) with HST/ Faint Object Spectrograph (FOS), or in our own survey of lowmass multiplicity with Keck laser guide star AO (LGSAO; A. Kraus et al. 2009, in preparation). As before, we inferred NIR magnitudes from the total system fluxes reported in 2MASS and spatially resolved flux ratios reported by Leinert et al. (1993), White \& Ghez (2001), Correia et al. (2006), and our LGSAO survey.

There are a small number of double-lined spectroscopic binaries that have been identified and studied in some detail in Taurus. We have added the well-known system UZ Tau Aab to our sample, adopting the spectral types and inferred $H$ band flux ratio found by Prato et al. (2002). We also added the short-period spectroscopic binary V773 Tau Aab, which was studied with RV and interferometric monitoring by Boden et al. (2007) as part of an orbit-monitoring program. The V773 Tau system also includes two faint companions at small separations and a wide brown dwarf companion; we do not include the close companions because they do not have spectral type determinations and because they are too faint to influence the observed properties of V773 Tau Aab (Duchêne et al. 2003), but we include the wide substellar companion (Luhman 2004). We also considered whether to include V826 Tau (Massarotti et al. 2005) and DQ Tau (Mathieu et al. 1997), but the only known flux ratio for V826 Tau is in the optical and there are no flux ratios reported for DQ Tau, so these systems could not be integrated with the rest of our sample.

We also note that one binary system in our sample, XZ Tau, was recently suggested to be a possible hierarchical triple based on resolved radio observations of XZ Tau B at $1.3 \mathrm{~cm}$ and $7 \mathrm{~mm}$. We have retained this system in our sample because the companion was not identified with HST (Krist et al. 1998) or in other surveys, but the suggested separation ( $~ 90$ mas or $13 \mathrm{AU}$ ) was near the detection limits of the earlier observations, so we regard it as a credible possibility. As we will show later, XZ Tau $\mathrm{B}$ is overluminous compared with XZ Tau A or HL Tau, which supports its probable multiplicity.

Finally, most of the binary components in our sample have spatially resolved photometry in the $K$ filter only. Inferring the component luminosities from such a red bandpass might introduce systematic errors in our estimated luminosities due to near-infrared excesses from circumstellar disks. In order to address this prospect, we have searched the literature to determine which stars are likely to host a warm disk; we summarize our assessments and the corresponding references in Table 1. We based these assessments, in order of priority, on 3-10 $\mu \mathrm{m}$ photometry (from Spitzer/Infrared Array Camera (IRAC) or ground-based AO imaging), optical spectroscopic accretion signatures, 10-30 $\mu \mathrm{m}$ spectroscopy (from Spitzer/ IRS), and finally on submillimeter/millimeter photometry. In each case where sufficient data are available, we have concluded that the star either has a disk ("Y"), does not have a disk ("N"), or might have a disk (but the observations are not spatially resolved, so we cannot determine which binary component(s) have one; "Y?"). We will address the significance of flux excesses from warm dust in Section 5.

\section{ANALYSIS}

\subsection{Inferred and Calculated Stellar Properties}

Any comparison of observations to stellar evolutionary models requires the conversion of observed properties (spectral types, filtered magnitudes, and extinctions) into fundamental physical parameters (effective temperatures and bolometric luminosities). This process is accomplished by invoking a temperature scale, a set of bolometric corrections, a reddening law, and an estimated distance. Temperature scales directly relate 
Table 1

Binary Sample: Observed and Inferred Properties

\begin{tabular}{|c|c|c|c|c|c|c|c|c|c|c|}
\hline Name & \multicolumn{2}{|c|}{$(\mathrm{J} 2000)$} & $\begin{array}{c}\text { Sep } \\
\text { (mas) }\end{array}$ & $\begin{array}{c}\text { Flux } \\
\text { (mag) }\end{array}$ & $\mathrm{SpT}$ & $\begin{array}{c}A_{V} \\
(\mathrm{mag})\end{array}$ & $\begin{array}{l}T_{\text {eff }} \\
(\mathrm{K}) \\
\end{array}$ & $\begin{array}{c}M_{\mathrm{bol}} \\
(\mathrm{mag})\end{array}$ & $\begin{array}{l}\text { Warm } \\
\text { Disk? }\end{array}$ & Refs \\
\hline HBC 352 & 35429.51 & +320301.4 & $8970 \pm 70$ & $J=10.09$ & $\mathrm{G} 0 \pm 2$ & 0.9 & $6030 \pm 170$ & 4.99 & $\ldots$ & 1,2 \\
\hline HBC 353 & 35430.17 & +320304.3 & $8970 \pm 70$ & $J=10.45$ & $\mathrm{G} 5 \pm 2$ & 1.0 & $5770 \pm 100$ & 5.41 & $\ldots$ & 1,2 \\
\hline НВС 355 & 35435.97 & +253708.1 & $6310 \pm 70$ & $J=10.81$ & $\mathrm{~K} 0 \pm 2$ & 0.5 & $5250 \pm 335$ & 6.08 & $\ldots$ & 1,3 \\
\hline HBC 354 & 35435.56 & +253711.1 & $6310 \pm 70$ & $J=11.80$ & $\mathrm{~K} 3 \pm 1$ & 1.2 & $4750 \pm 155$ & 7.05 & $\ldots$ & 1,3 \\
\hline НBC 356 & 40313.96 & +255259.8 & $1280 \pm 20$ & $J=10.84$ & $\mathrm{~K} 3 \pm 1$ & 0.7 & $4750 \pm 155$ & 6.23 & $\mathrm{~N}$ & $4,5,20$ \\
\hline НBC 357 & 40313.96 & +255259.8 & $1280 \pm 20$ & $J=10.84$ & $\mathrm{~K} 3 \pm 1$ & 0.7 & $4750 \pm 155$ & 6.23 & $\mathrm{~N}$ & $4,5,20$ \\
\hline V773 Tau Aa & 41412.92 & +281212.4 & $S B$ & $K=6.72$ & $\mathrm{~K} 2 \pm 1$ & 1.8 & $4900 \pm 150$ & 2.56 & $\mathrm{Y} ?$ & 6,20 \\
\hline V773 Tau Ab & 41412.92 & +281212.4 & $S B$ & $K=7.27$ & $\mathrm{~K} 3 \pm 1.5$ & 1.8 & $4750 \pm 200$ & 3.25 & $Y ?$ & 6,20 \\
\hline 2M04141188 & 41411.88 & +281153.5 & $23380 \pm 70$ & $J=13.16$ & $\mathrm{M} 6.25 \pm 0.25$ & 1.0 & $2960 \pm 30$ & 9.00 & $\ldots$ & 3,7 \\
\hline FO Tau A & 41449.29 & +281230.6 & $152.5 \pm 2.9$ & $K=8.87$ & $\mathrm{M} 3.5 \pm 0.5$ & 1.9 & $3340 \pm 75$ & 5.53 & $\mathrm{Y}$ & 8,9 \\
\hline FO Tau B & 41449.29 & +281230.6 & $152.5 \pm 2.9$ & $K=8.87$ & $\mathrm{M} 3.5 \pm 0.5$ & 1.9 & $3340 \pm 75$ & 5.53 & $\mathrm{Y}$ & 8,9 \\
\hline DD Tau A & 41831.13 & +281629.0 & $555 \pm 10$ & $K=8.45$ & $\mathrm{M} 3.5 \pm 0.5$ & 2.1 & $3340 \pm 75$ & 5.08 & $\mathrm{Y}$ & $8,9,21$ \\
\hline DD Tau B & 41831.13 & +281629.0 & $555 \pm 10$ & $K=8.85$ & $\mathrm{M} 3.5 \pm 0.5$ & 2.9 & $3340 \pm 75$ & 5.40 & $\mathrm{Y}$ & $8,9,21$ \\
\hline FQ Tau A & 41912.81 & +282933.1 & $752 \pm 14$ & $K=10.03$ & $\mathrm{M} 3 \pm 0.5$ & 2.0 & $3415 \pm 75$ & 6.66 & $\mathrm{Y}$ & $8,9,21$ \\
\hline FQ Tau B & 41912.81 & +282933.1 & $752 \pm 14$ & $K=10.11$ & $\mathrm{M} 3.5 \pm 0.5$ & 1.8 & $3340 \pm 75$ & 6.78 & $\mathrm{Y}$ & $8,9,21$ \\
\hline LkCa 7 A & 41941.27 & +274948.5 & $1021 \pm 19$ & $K=8.74$ & $\mathrm{M} 0 \pm 0.5$ & 0.2 & $3850 \pm 90$ & 5.34 & $\mathrm{~N}$ & $8,9,20$ \\
\hline $\mathrm{LkCa} 7 \mathrm{~B}$ & 41941.27 & +274948.5 & $1021 \pm 19$ & $K=9.37$ & $\mathrm{M} 3.5 \pm 0.5$ & 0.4 & $3340 \pm 75$ & 6.20 & $\mathrm{~N}$ & $8,9,20$ \\
\hline FS Tau A & 42202.18 & +265730.5 & $227.6 \pm 7.1$ & $K=8.33$ & $\mathrm{M} 0 \pm 0.5$ & 5.0 & $3850 \pm 90$ & 4.40 & $\mathrm{Y}$ & 8,9 \\
\hline FS Tau B & 42202.18 & +265730.5 & $227.6 \pm 7.1$ & $K=10.43$ & $\mathrm{M} 3.5 \pm 0.5$ & 5.2 & $3340 \pm 75$ & 6.72 & $\mathrm{Y}$ & 8,9 \\
\hline Haro 6-5B & 42200.69 & +265733.3 & $19880 \pm 70$ & $J=15.08$ & $\mathrm{~K} 5 \pm 2$ & 10.0 & $4350 \pm 450$ & 8.01 & $\mathrm{Y}$ & 10,11 \\
\hline FV Tau A & 42653.53 & +260654.4 & $12081 \pm 9$ & $J=9.92$ & $\mathrm{~K} 5 \pm 0.5$ & 5.4 & $4350 \pm 135$ & 4.11 & $\mathrm{Y}$ & $8,9,21$ \\
\hline FV Tau/c A & 42654.41 & +260651.0 & $12081 \pm 9$ & $K=9.00$ & $\mathrm{M} 2.5 \pm 0.5$ & 3.3 & $3485 \pm 75$ & 5.46 & $\mathrm{~N}$ & $8,9,21$ \\
\hline FV Tau/c B & 42654.41 & +260651.0 & $713 \pm 1.8$ & $K=11.21$ & $\mathrm{M} 3.5 \pm 0.5$ & 7.0 & $3340 \pm 75$ & 7.30 & $\mathrm{Y}$ & $8,9,21$ \\
\hline DF Tau A & 42702.80 & +254222.3 & $103 \pm 2$ & $K=7.13$ & $\mathrm{M} 2 \pm 0.5$ & 0.6 & $3560 \pm 75$ & 3.84 & $\mathrm{Y}$ & 5,8 \\
\hline DF Tau B & 42702.80 & +254222.3 & $103 \pm 2$ & $K=8.01$ & $\mathrm{M} 2.5 \pm 0.5$ & 0.8 & $3485 \pm 75$ & 4.75 & $\mathrm{Y}$ & 5,8 \\
\hline 2M04284263 A & 42842.63 & +271403.9 & $621 \pm 7$ & $K=10.85$ & $\mathrm{M} 5 \pm 0.5$ & 0.5 & $3125 \pm 75$ & 7.84 & Y? & $7,12,23$ \\
\hline 2M04284263 B & 42842.63 & +271403.9 & $621 \pm 7$ & $K=11.75$ & M5.5 \pm 0.5 & 0.5 & $3055 \pm 70$ & 8.76 & Y? & $3,7,12,23$ \\
\hline UX Tau A & 43004.00 & +181349.4 & $5856 \pm 3$ & $K=7.60$ & $\mathrm{~K} 2 \pm 1$ & 0.2 & $4900 \pm 165$ & 3.62 & $\mathrm{Y}$ & $1,13,21$ \\
\hline UX Tau C & 43004.00 & +18 1349.4 & $2692 \pm 2$ & $K=10.85$ & $\mathrm{M} 5 \pm 0.5$ & 0.1 & $3125 \pm 75$ & 7.88 & $\mathrm{~N}$ & $1,13,14,21$ \\
\hline FX Tau A & 43029.61 & +242645.0 & $890 \pm 17$ & $K=8.33$ & $\mathrm{M} 1 \pm 1$ & 1.1 & $3705 \pm 145$ & 4.91 & $\mathrm{Y}$ & $4,9,21$ \\
\hline FX Tau B & 43029.61 & +242645.0 & $890 \pm 17$ & $K=9.19$ & $\mathrm{M} 4 \pm 1$ & 1.1 & $3270 \pm 145$ & 5.97 & $\mathrm{~N}$ & $4,9,21$ \\
\hline DK Tau A & 43044.25 & +260124.5 & $2360 \pm 1$ & $K=7.36$ & $\mathrm{~K} 9 \pm 1$ & 0.8 & $4060 \pm 250$ & 3.66 & Y? & $4,13,22$ \\
\hline DK Tau B & 43044.25 & +260124.5 & $2360 \pm 1$ & $K=8.74$ & $\mathrm{M} 1 \pm 1$ & 0.8 & $3705 \pm 145$ & 5.35 & Y? & $4,13,22$ \\
\hline V927 Tau A & 43123.82 & +241052.9 & $267 \pm 6.8$ & $K=9.31$ & $\mathrm{M} 3 \pm 0.5$ & 1.4 & $3415 \pm 75$ & 6.00 & $\mathrm{~N}$ & $8,9,23$ \\
\hline V927 Tau B & 43123.82 & +241052.9 & $267 \pm 6.8$ & $K=9.79$ & $\mathrm{M} 3.5 \pm 0.5$ & 0.9 & $3340 \pm 75$ & 6.56 & $\mathrm{~N}$ & $8,9,23$ \\
\hline HL Tau & 43138.44 & +181357.7 & $23310 \pm 70$ & $J=10.62$ & $\mathrm{~K} 5 \pm 1$ & 7.4 & $4350 \pm 265$ & 4.25 & $\mathrm{Y}$ & 10,15 \\
\hline XZ Tau A & 43140.07 & +181357.2 & $300.6 \pm 1.3$ & $K=8.36$ & $\mathrm{M} 2 \pm 1$ & 1.4 & $3560 \pm 145$ & 4.98 & $\mathrm{Y}$ & 8,9 \\
\hline XZ Tau B & 43140.07 & +181357.2 & $300.6 \pm 1.3$ & $K=7.80$ & $\mathrm{M} 3.5 \pm 0.5$ & 1.4 & $3340 \pm 75$ & 4.52 & $\mathrm{Y}$ & 8,9 \\
\hline HK Tau A & 43150.57 & +242418.1 & $2342 \pm 61$ & $K=8.64$ & $\mathrm{M} 1 \pm 0.5$ & 2.3 & $3705 \pm 75$ & 5.08 & $\mathrm{Y}$ & $1,9,10,23$ \\
\hline HK Tau B & 43150.57 & +242418.1 & $2342 \pm 61$ & $K=11.96$ & $\mathrm{M} 1 \pm 0.5$ & 2.3 & $3705 \pm 75$ & 8.40 & $\mathrm{Y}$ & $1,9,10$ \\
\hline V710 Tau A & 43157.79 & +182138.1 & $3224 \pm 3$ & $K=9.38$ & $\mathrm{M} 1 \pm 1$ & 0.9 & $3705 \pm 145$ & 5.98 & $\mathrm{Y}$ & $1,13,21$ \\
\hline V710 Tau B & 43157.79 & +182138.1 & $3224 \pm 3$ & $K=9.44$ & $\mathrm{M} 3 \pm 1$ & 0.9 & $3415 \pm 145$ & 6.19 & $\mathrm{~N}$ & $1,13,21$ \\
\hline V710 Tau C & 43157.79 & +182138.1 & $28000 \pm 70$ & $J=12.26$ & $\mathrm{M} 3 \pm 0.5$ & 0.9 & $3415 \pm 75$ & 8.00 & $\mathrm{Y}$ & $1,3,16$ \\
\hline GG Tau Aa & 43230.35 & +173140.6 & $10100 \pm 7$ & $J=9.07$ & $\mathrm{~K} 7 \pm 1$ & 0.7 & $4060 \pm 250$ & 4.50 & $\mathrm{Y}$ & $13,17,21$ \\
\hline GG Tau Ab & 43230.35 & +173140.6 & $250.2 \pm 2.6$ & $J=9.95$ & $\mathrm{M} 0.5 \pm 0.5$ & 3.2 & $3775 \pm 75$ & 4.87 & $\mathrm{Y}$ & $13,17,21$ \\
\hline GG Tau Ba & 43230.35 & +173140.6 & $10100 \pm 7$ & $J=11.28$ & $\mathrm{M} 6 \pm 0.5$ & 0.6 & $2990 \pm 65$ & 7.24 & $\mathrm{Y}$ & $13,14,17,21$ \\
\hline GG Tau Bb & 43230.35 & +173140.6 & $1476.5 \pm 6.5$ & $J=12.96$ & $\mathrm{M} 7.5 \pm 0.5$ & 0.0 & $2795 \pm 85$ & 9.10 & $\mathrm{Y}$ & $13,14,17,21$ \\
\hline UZ Tau Aa & 43243.04 & +255231.1 & $S B$ & $H=8.54$ & $\mathrm{M} 1 \pm 1$ & 1.5 & $3705 \pm 145$ & 4.77 & $\mathrm{Y}$ & $1,18,22$ \\
\hline UZ Tau Ab & 43243.04 & +255231.1 & $S B$ & $H=9.36$ & $\mathrm{M} 4 \pm 1$ & 1.5 & $3270 \pm 145$ & 5.73 & $\mathrm{Y}$ & $1,18,22$ \\
\hline UZ Tau Ba & 43243.04 & +255231.1 & $3539.5 \pm 2.1$ & $H=8.46$ & $\mathrm{M} 2 \pm 0.5$ & 0.6 & $3560 \pm 75$ & 4.91 & $\mathrm{Y}$ & $8,9,21$ \\
\hline UZ Tau Bb & 43243.04 & +255231.1 & $367.8 \pm 1$ & $H=9.18$ & $\mathrm{M} 3 \pm 0.5$ & 1.8 & $3415 \pm 75$ & 5.48 & $\mathrm{Y}$ & $8,9,21$ \\
\hline GH Tau A & 43306.22 & +240934.0 & $311.1 \pm 1.3$ & $K=8.66$ & $\mathrm{M} 2 \pm 0.5$ & 0.0 & $3560 \pm 75$ & 5.44 & $\mathrm{Y}$ & $8,9,21$ \\
\hline GH Tau B & 43306.22 & +240934.0 & $311.1 \pm 1.3$ & $K=8.45$ & $\mathrm{M} 2 \pm 0.5$ & 0.5 & $3560 \pm 75$ & 5.17 & $\mathrm{Y}$ & $8,9,21$ \\
\hline IS Tau A & 43336.79 & +260949.2 & $222.8 \pm 2.4$ & $K=8.82$ & $\mathrm{M} 0 \pm 0.5$ & 3.3 & $3850 \pm 90$ & 5.08 & $\mathrm{Y}$ & 8,9 \\
\hline IS Tau B & 43336.79 & +260949.2 & $222.8 \pm 2.4$ & $K=10.72$ & $\mathrm{M} 3.5 \pm 0.5$ & 3.6 & $3340 \pm 75$ & 7.19 & $\mathrm{~N}$ & 8,9 \\
\hline HN Tau A & 43339.35 & +175152.4 & $3142 \pm 1$ & $K=8.51$ & $\mathrm{~K} 5 \pm 1$ & 0.5 & $4350 \pm 265$ & 4.82 & Y? & $1,13,20$ \\
\hline HN Tau B & 43339.35 & +175152.4 & $3142 \pm 1$ & $K=10.81$ & $\mathrm{M} 4.5 \pm 1$ & 0.5 & $3200 \pm 180$ & 7.72 & Y? & $4,13,20$ \\
\hline IT Tau A & 43354.70 & +261327.5 & $2416 \pm 8$ & $K=8.12$ & $\mathrm{~K} 3 \pm 1$ & 4.1 & $4750 \pm 155$ & 3.84 & Y? & $4,13,22$ \\
\hline IT Tau B & 43354.70 & +261327.5 & $2416 \pm 8$ & $K=9.54$ & $\mathrm{M} 4 \pm 1$ & 4.1 & $3270 \pm 145$ & 5.98 & Y? & $4,13,22$ \\
\hline Haro 6-28 A & 43556.84 & +225436.0 & $647 \pm 12$ & $K=10.12$ & $\mathrm{M} 2 \pm 0.5$ & 2.3 & $3560 \pm 75$ & 6.64 & $\mathrm{Y} ?$ & $8,9,24$ \\
\hline Haro 6-28 B & 43556.84 & +225436.0 & $647 \pm 12$ & $K=10.48$ & $\mathrm{M} 3.5 \pm 0.5$ & 1.9 & $3340 \pm 75$ & 7.14 & Y? & $8,9,24$ \\
\hline 2M04414565 & 44145.65 & +230158.0 & $12370 \pm 70$ & $J=10.74$ & $\mathrm{M} 3 \pm 0.5$ & 0.0 & $3415 \pm 75$ & 6.72 & $\ldots$ & 3,16 \\
\hline 2M04414489 & 44144.89 & +230151.3 & $12370 \pm 70$ & $J=14.42$ & $\mathrm{M} 8.25 \pm 0.25$ & 0.0 & $2630 \pm 78$ & 10.56 & $\ldots$ & $3,16,19$ \\
\hline LkHa332-G2 A & 44207.33 & +252303.2 & $234.1 \pm 4.5$ & $K=8.38$ & $\mathrm{M} 0.5 \pm 0.5$ & 2.0 & $3775 \pm 75$ & 4.82 & $\mathrm{~N}$ & $8,9,22$ \\
\hline
\end{tabular}


Table 1

(Continued)

\begin{tabular}{|c|c|c|c|c|c|c|c|c|c|c|}
\hline Name & R.A. & 00) Decl. & $\begin{array}{c}\text { Sep } \\
\text { (mas) }\end{array}$ & $\begin{array}{l}\text { Flux } \\
(\operatorname{mag})\end{array}$ & $\mathrm{SpT}$ & $\begin{array}{c}A_{V} \\
(\mathrm{mag})\end{array}$ & $\begin{array}{l}T_{\text {eff }} \\
(\mathrm{K})\end{array}$ & $\begin{array}{c}M_{\mathrm{bol}} \\
(\mathrm{mag})\end{array}$ & $\begin{array}{l}\text { Warm } \\
\text { Disk? }\end{array}$ & Refs \\
\hline LkHa332-G2 B & 44207.33 & +252303.2 & $234.1 \pm 4.5$ & $K=9.16$ & $\mathrm{M} 2.5 \pm 0.5$ & 3.3 & $3485 \pm 75$ & 5.61 & $\mathrm{~N}$ & $8,9,22$ \\
\hline V955 Tau A & 44207.77 & +252311.8 & $330.9 \pm 1.2$ & $K=8.18$ & $\mathrm{~K} 7 \pm 0.5$ & 2.8 & $4060 \pm 125$ & 4.26 & $\mathrm{Y}$ & $8,9,21$ \\
\hline V955 Tau B & 44207.77 & +252311.8 & $330.9 \pm 1.2$ & $K=9.72$ & $\mathrm{M} 2.5 \pm 0.5$ & 2.3 & $3485 \pm 75$ & 6.28 & $\mathrm{Y}$ & $8,9,21$ \\
\hline UY Aur A & 45147.38 & +304713.5 & $878 \pm 17$ & $K=7.68$ & $\mathrm{M} 0 \pm 0.5$ & 0.6 & $3850 \pm 90$ & 4.24 & $\mathrm{Y}$ & 8,9 \\
\hline UY Aur B & 45147.38 & +304713.5 & $878 \pm 17$ & $K=8.44$ & $\mathrm{M} 2.5 \pm 0.5$ & 2.7 & $3485 \pm 75$ & 4.96 & $\mathrm{Y}$ & 8,9 \\
\hline 2M04554757 & 45547.57 & +302807.7 & $6310 \pm 70$ & $J=11.05$ & $\mathrm{M} 4.75 \pm 0.25$ & 0.0 & $3165 \pm 50$ & 7.15 & $\ldots$ & 3,7 \\
\hline 2M04554801 & 45548.01 & +302805.0 & $6310 \pm 70$ & $J=13.19$ & M5.6 \pm 0.25 & 0.0 & $3045 \pm 35$ & 9.31 & $\ldots$ & 3,7 \\
\hline RW Aur A & 50749.54 & +302405.1 & $1417.5 \pm 3.4$ & $K=7.25$ & $\mathrm{~K} 2 \pm 2$ & 1.6 & $4900 \pm 330$ & 3.11 & $\mathrm{Y}$ & $9,10,21$ \\
\hline RW Aur B & 50749.54 & +302405.1 & $1417.5 \pm 3.4$ & $K=8.82$ & $\mathrm{~K} 6 \pm 1$ & 1.6 & $4350 \pm 265$ & 5.01 & $\mathrm{Y}$ & $9,10,21$ \\
\hline
\end{tabular}

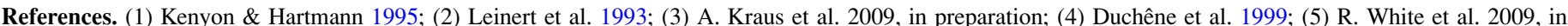

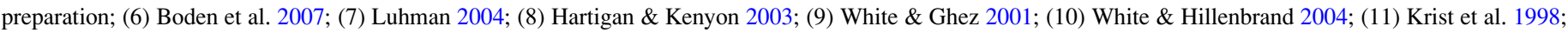

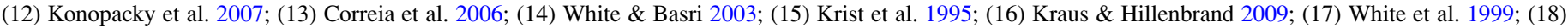

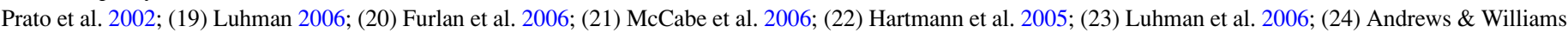
2005.

spectral types to temperatures, allowing the estimation of temperatures based on observed spectral features. Temperatures are also used to define intrinsic colors, from which an observed color can be used to infer the reddening and extinction. Bolometric corrections are temperature-dependent ratios of the flux in a filtered band to the full bolometric flux and are calibrated for nearby field stars that have been studied across the full range of wavelengths with a significant contribution to the luminosity. Once the bolometric flux is known, the distance for a star then directly yields the bolometric luminosity.

Observed spectral types can be converted into effective temperatures using an adopted temperature scale, but this process is somewhat uncertain for young stars since the relation between temperature and spectral type is sensitive to surface gravity. For M-type stars, a giant with a given spectral type can be $\sim 300 \mathrm{~K}$ warmer than a dwarf of the same type (e.g., Leggett et al. 1996 versus Perrin et al. 1998; Richichi et al. 1998; van Belle et al. 1999). Young stars have intermediate surface gravity, so it has been suggested (e.g., Luhman et al. 1997; Luhman et al. 2003) that an intermediate temperature scale might be appropriate. For example, Luhman et al. (2003) found that M2M5 stars in IC 348 have bluer $R-I$ colors than their field counterparts, matching the trend seen for giants. Similarly, we have shown in a previous publication (Kraus et al. 2006) that the $V-I$ colors of M5-M9 stars in Taurus and Upper Sco are significantly bluer than those of field dwarfs, though not as blue as for giants. Conversely, more recent surveys of moderately older stars in $\epsilon$ Cha and $\eta$ Cha by Lyo et al. $(2004,2008)$ show that the broadband colors and temperature-sensitive narrowband spectral indices are generally similar to field dwarfs to spectral types as late as M5.5, with only a mild discrepancy in the $B-$ $V$ color that could be attributed to chromospheric activity.

We have adopted the temperature scale suggested by Luhman et al. (2003) for use in low-gravity young stars. For spectral types $\lesssim \mathrm{M} 0$, the temperature-SpT relation does not appear to be gravity sensitive, so Luhman et al. use the temperature scale of Schmidt-Kaler (1982). For late-type ( $\gtrsim$ M0) young stars, Luhman et al. define an intermediate temperature scale that makes the average cluster sequences of Taurus and IC348 internally coeval with respect to the NextGen models (Baraffe et al. 1998). Unfortunately, this calibration introduces an element of circularity into our analysis; the temperature scale is chosen by definition to make our chosen models reproduce observations. It is unclear whether the temperature scale of young stars is truly different or the adopted temperature scale is correcting for a discrepancy in the model, so our results should be weighed accordingly. The difference in inferred temperatures with respect to field $\mathrm{M}$ dwarf values (Leggett et al. 1996) is $\sim 50 \mathrm{~K}$ at M3, $\sim 100 \mathrm{~K}$ at M5, and $\sim 200 \mathrm{~K}$ at M8, equivalent to a systematic uncertainty of $\pm 0.25, \pm 0.5$, and \pm 0.75 subclass, respectively.

We have adopted the bolometric corrections that we previously described for use with field stars (Kraus \& Hillenbrand $2007 b$ ). For spectral types $\lesssim K 7$, we used the corrections suggested by Masana et al. (2006), while for M dwarfs, we used the corrections of Leggett (1992) and Leggett et al. (1996). Values calibrated for field dwarfs might be systematically inaccurate if the bolometric correction is also gravity sensitive, so it would be helpful to verify whether these bolometric corrections are valid for young stars. However, we are not aware of any such tests having been attempted. In all cases, we have adopted the observed NIR flux (and its bolometric correction) that is closest to the $J$ filter. As we describe below, luminosity excesses are typically least significant in the $I$ and $J$ filters, but most of the binary systems in our sample have resolved flux measurements only in the $H$ or $K$ filter.

We have implemented our extinction corrections using the interstellar reddening law of Schlegel et al. (1998), which stipulates that one magnitude of visual extinction corresponds to $A_{J}=0.28, A_{H}=0.18$, and $A_{K}=0.11$; these values are consistent with the interstellar reddening law in the 2MASS filters suggested by Indebetouw et al. (2005). Reddening laws might vary in regions with extremely high density (Weingartner \& Draine 2001; Román-Zúñiga et al. 2007), but most of our sample members are only moderately reddened. An analysis of 2MASS source counts and colors toward the Ophiuchus, Lupus, and Pipe Nebulae suggests that the standard interstellar reddening law is appropriate to extinctions of $A_{V} \sim 20$ or more (Lombardi et al. 2008).

We adopted a characteristic distance for all Taurus members of $145 \pm 15 \mathrm{pc}$. Recent high-precision parallax measurements with the VLBA (Lestrade et al. 1999; Loinard et al. 2007, 2008; Torres et al. 2007) suggest that there might be a distance gradient of $165-125 \mathrm{pc}$ in the east-west direction, though the discrepant distances of neighboring V773 Tau and Hubble 4 (148 \pm 5 pc versus $132.5 \pm 0.6 \mathrm{pc}$ ) suggest an overall scatter at any location of $\sim 10-15 \mathrm{pc}(10 \%)$. The luminosity uncertainty, if we adopt the characteristic distance, is only $\sim 0.1-0.2 \mathrm{mag}$, which is similar to the uncertainty from dereddening and intrinsic 
variability, so attempting to extrapolate more precise distances from this suggestion of three-dimensional structure is not likely to improve our results.

We should note that there is room for a significant uncertainty in our inferred luminosities due to the intrinsic variability of young stars. Variability in Class III stars should be caused by cool spots, so its characteristic amplitude at near-infrared wavelengths should be not more than $\sim 0.1 \mathrm{mag}$ (e.g., Carpenter et al. 2001, 2002). However, the same survey showed that Class I-II stars occasionally vary by as much as $1-2$ mag in the near infrared, and extreme classes of stars (such as FUor and EXor stars) can vary by even more. This suggests that an unusually young apparent age for one binary component could be the result of variability in that component.

A star that is surrounded by circumstellar material could also appear systematically underluminous by several magnitudes if it possesses an edge-on disk or a massive circumstellar envelope that renders it visible only in scattered light. It would then appear much older than its unobscured companion. One such case is HK Tau B; both components of this system have a spectral type of M1 (White \& Hillenbrand 2004), but the B component is 3.3 magnitudes fainter in $K$.

Finally, the presence of veiling due to accretion (at blue wavelengths) or circumstellar dust emission (at red wavelengths) could bias the inferred luminosity of a binary component. Observations in the $I$ or $J$ filter are least sensitive to this veiling, though even those measurements can be significantly impacted (Folha \& Emerson 1999; White \& Hillenbrand 2004). In our study, we have used photometry from $J$ or from the nearest redder filter. We would prefer to use $J$ for all systems, but in most cases, the absence of suitable data forces us to use relative photometry in $H$ or $K$. Data from bluer filters are also available for some sample members, but these data are not as homogeneous and we prefer to minimize the total wavelength range over which we make our luminosity estimates.

We list the stellar properties that we inferred with these methods in Table 1. Our temperature uncertainties have been determined from the uncertainty in each star's spectral type. Each of the bolometric magnitude uncertainties listed above should contribute $\sim 0.1 \mathrm{mag}$, so we have adopted a total statistical uncertainty of $0.3 \mathrm{mag}$. However, many of the uncertainties (such as for distance and extinction) should be correlated between binary components, so the uncertainty in their relative ages should be lower than our formal estimates. As we discuss further in Section 5, there is compelling evidence that the scatter in relative ages of binary components is indeed significantly smaller, suggesting that these error estimates are conservative.

\subsection{Inferred Physical Stellar Parameters}

Several sets of pre-main sequence evolutionary models have been developed in recent decades, but all of these models still face significant challenges in confronting observational constraints. Hillenbrand \& White (2004) found that all models have difficulty matching the dynamical masses of young stars with $M \lesssim 1.2 M_{\odot}$, a range that encompasses almost all of our sample. However, the Lyon models (Baraffe et al. 1998; Chabrier et al. 2000) seem to work best for low-mass stars, especially when using a mixing length of $\alpha=1.0$ and the revised temperature scale of Luhman et al. (2003). All models were found to reproduce the observed masses for stars with $M \gtrsim 1.2 M_{\odot}$, though the Lyon models only extend to $1.4 M_{\odot}$.

In light of these results, we have adopted a hybrid combination of the Lyon models for low-mass stars and the models of
D’Antona \& Mazzitelli (1997, hereafter DM97) for higher mass stars. For masses $\leqslant 0.5 M_{\odot}$, we use the mass-luminositytemperature relations of the Lyon models with a mixing length of $\alpha=1.0$. For masses $\geqslant 1.0 M_{\odot}$, we use the corresponding relations of DM97. Finally, in the intermediate regime of 0.6$0.9 M_{\odot}$, we adopt a weighted average of the luminosity and temperature predicted by each model in order to produce a smooth transition between the two sets of models. This choice does not provide any insight into the missing physics that are still required to bring the models into agreement, but it represents an acceptable compromise for estimating relative ages of a sample of young stars. The two sets of models converge at older ages, so our solution is only important for very young stars $(\lesssim 10$ Myr $)$.

These models report stellar luminosities and temperatures at quantized values of age and mass, so for each of our sample members, we have linearly interpolated between the four values of $T_{\text {eff }}$ and $M_{\text {bol }}$ around it in the HR diagram. The Lyon models also face a significant challenge with respect to very young stars since they are not defined for ages of $<1 \mathrm{Myr}$, so for each star that falls above this isochrone and has a mass within the affected range $\left(<1 M_{\odot}\right)$, we have linearly extrapolated its age from the four points below it in the HR diagram. These extrapolated ages should be regarded as much more uncertain than older ages, but the degree of error should be similar for stars with similar HR diagram positions, so only systems with disparate masses will be subject to the full systematic uncertainty.

In Table 2, we list the inferred mass and age for each star from our hybrid system and from the default Lyon and DM97 models. Several sample members illustrate the extreme difference in mass and age estimates for the two sets of models. For example, the inferred parameters of $\mathrm{HN}$ Tau A are $M=1.35 M_{\odot}$ and $\log (\tau)=6.85$ according to the Lyon models and $M=0.65 M_{\odot}$ and $\log (\tau)=6.05$ according to the DM97 models; our hybrid isochrones yield $M=0.85 M_{\odot}$ and $\log (\tau)=6.27$. The uncertainty in $\log (\tau)$ due to observational errors is \pm 0.25 dex for an average star in our sample, but as we describe below, this uncertainty could be an overestimate since binary systems appear to be more coeval.

\section{THE HR DIAGRAM}

The overall population sequence in an HR diagram provides a valuable test of our choice of evolutionary models. If the association is nominally coeval, then it should trace a single recognizable sequence that is parallel to theoretical isochrones. The individual sample members that strongly deviate from the association sequence should also be examined for a systematic source of error such as erroneous membership, circumstellar material that blocks and scatters the stellar flux, or misclassification of spectral types.

In Figure 1, we show the HR diagram for all of our binary sample members. The cluster sequence seems to trace the 1-2 Myr isochrone, albeit with significant scatter due to the many sources of uncertainty and the unknown spread of stellar ages. We adopted the two sets of theoretical isochrones (Lyon for low-mass stars and DM97 for higher mass stars) specifically because they fit the Taurus single-star sequence across the full mass range of our sample (Appendix A), so this agreement with the canonical age of Taurus is not surprising. However, many individual members fall unusually low; as we show in Figure A2 for single stars, the $5 \mathrm{Myr}$ isochrone represents a $+2 \sigma$ deviation from the median age of Taurus, so we adopt this approximate lower limit for the observed scatter of the main body of members. 
Table 2

Derived Ages and Masses

\begin{tabular}{|c|c|c|c|c|c|c|}
\hline Name & $M\left(M_{\odot}\right)$ & (Adopted) & $M\left(M_{\odot}\right)$ & $\log (\tau)(\mathrm{yr})$ & \multicolumn{2}{|c|}{ (DM97) } \\
\hline HBC 352 & $\ldots$ & $>7.7$ & $\ldots$ & $>7.7$ & $\ldots$ & $>7.7$ \\
\hline HBC 353 & $\ldots$ & $>7.7$ & $\ldots$ & $>7.7$ & $\ldots$ & $>7.7$ \\
\hline HBC 355 & $\ldots$ & $>7.7$ & $\ldots$ & $>7.7$ & $\ldots$ & $>7.7$ \\
\hline HBC 354 & $\ldots$ & $>7.7$ & $\ldots$ & $>7.7$ & $\ldots$ & $>7.7$ \\
\hline HBC 356 & $0.79_{-0.03}^{+0.08}$ & $7.68_{-0.27}^{+0.65}$ & $\ldots$ & $>7.7$ & $0.79_{-0.05}^{+0.09}$ & $7.56_{-0.27}^{+0.52}$ \\
\hline HBC 357 & $0.79_{-0.03}^{+0.08}$ & $7.68_{-0.27}^{+0.65}$ & $\ldots$ & $>7.7$ & $\begin{array}{l}-0.05 \\
0.79_{-0.05}^{+0.09}\end{array}$ & $\begin{array}{l}-0.27 \\
7.56_{-0.27}^{+0.52}\end{array}$ \\
\hline V773 Tau Aa & $1.5_{-0.3}^{+0.4}$ & $5.61_{-0.21}^{-0.22}$ & $1.9_{-0.2}^{+0.3}$ & $5.92_{-0.27}^{+0.28}$ & $1.5_{-0.3}^{+0.4}$ & $5.61_{-0.21}^{+0.22}$ \\
\hline V773 Tau Ab & $1.0_{-0.1}^{+0.5}$ & $5.62_{-0.24}^{+0.41}$ & $1.9_{-0.3}^{+0.3}$ & $6.25_{-0.31}^{+0.48}$ & $1.1_{-0.4}^{+0.4}$ & $5.69_{-0.35}^{+0.31}$ \\
\hline $2 \mathrm{M} 04141188$ & $0.070_{-0.007}^{+0.008}$ & $6.46_{-0.19}^{+0.19}$ & $0.070_{-0.007}^{+0.008}$ & $6.46_{-0.19}^{+0.19}$ & $0.111_{-0.014}^{+0.013}$ & $6.83_{-012}^{+0.11}$ \\
\hline FO Tau A & $0.4^{+0.10}$ & $5.86^{+0.21}$ & $0.40^{+0.10}$ & $5.86^{+0.21}$ & $0.19^{+0.03}$ & $5.20^{-0.54}$ \\
\hline FO Tau B & $0.4_{-0.17}^{+0.10}$ & $5.86_{-0.28}^{+0.28}$ & $0.40_{-0.17}^{+0.10}$ & $5.86_{-0.28}^{+0.28}$ & $0.19_{-0.03}^{+0.03}$ & $5.20_{-0.27}^{+0.54}$ \\
\hline DD Tau A & $0.43_{-0.23}^{+0.17}$ & $5.63_{-0.32}^{+0.28}$ & $0.43_{-0.23}^{+0.13}$ & $5.63_{-0.32}^{+0.28}$ & $0.18_{-0.02}^{+0.03}$ & $4.85_{-0.25}^{-0.27}$ \\
\hline DD Tau B & $0.41_{-0.19}^{+0.11}$ & $5.79_{-0.29}^{+0.32}$ & $0.41_{-0.19}^{+0.11}$ & $5.79_{-0.29}^{-0.32}$ & $0.19_{-0.02}^{+0.02}$ & $5.09_{-0.26}^{+0.25}$ \\
\hline FQ Tau A & $0.37_{-0.05}^{+0.07}$ & $6.50_{-0.20}^{+0.23}$ & $0.37_{-0.05}^{+0.07}$ & $6.50_{-0.20}^{+0.23}$ & $0.25_{-0.03}^{+0.05}$ & $6.23_{-0.16}^{+0.20}$ \\
\hline FQ Tau B & $0.32_{-0.05}^{+0.05}$ & $6.43_{-0.19}^{+0.20}$ & $0.32_{-0.05}^{+0.05}$ & $6.43_{-0.19}^{+0.20}$ & $0.22_{-0.03}^{+0.03}$ & $6.21_{-0.15}^{+0.16}$ \\
\hline $\mathrm{LkCa} 7 \mathrm{~A}$ & $\begin{array}{l}0.67_{-0.04}^{+0.04} \\
0.04\end{array}$ & $6.28_{-0.21}^{+0.21}$ & $0.96_{-0.12}^{+0.05}$ & $6.61_{-022}^{+0.19}$ & $0.42_{-0.06}^{+0.03}$ & $\begin{array}{l}5.91_{-0.18}^{+0.153} \\
\end{array}$ \\
\hline $\mathrm{LkCa} 7 \mathrm{~B}$ & $0.35_{-0.07}^{-0.04}$ & $6.17_{-0.22}^{-0.17}$ & $0.35_{-0.07}^{-0.06}$ & $6.17_{-0.22}^{-0.22}$ & $0.21_{-0.03}^{+0.06}$ & $5.95_{-0.47}^{+0.18}$ \\
\hline FS Tau A & $\begin{array}{l}-0.07 \\
0.66_{-0.03}^{+0.03}\end{array}$ & $\begin{array}{l}-0.22 \\
5.67_{-0.20}^{+0.20}\end{array}$ & $\begin{array}{l}-0.07 \\
1.06_{-0.12}^{+0.10}\end{array}$ & $6.06_{-0.25}^{+0.22}$ & $\begin{array}{l}-0.03 \\
0.32_{-0.04}^{+0.05}\end{array}$ & $\begin{array}{r}-0.47 \\
5.16_{-0.39}^{+0.42}\end{array}$ \\
\hline FS Tau B & $0.32_{-0.05}^{+0.05}$ & $6.40_{-0.19}^{+0.20}$ & $0.32_{-0.05}^{+0.12}$ & $6.40_{-0.19}^{+0.20}$ & $0.22_{-0.03}^{+0.03}$ & $6.19_{-0.15}^{+0.17}$ \\
\hline Haro 6-5B & $\ldots$ & $>7.7$ & $\ldots$ & $>7.7$ & & $>7.7$ \\
\hline FV Tau A & $0.83_{-0.06}^{+0.06}$ & $5.85_{-018}^{+0.19}$ & $1.54^{+0.15}$ & $6.38_{-022}^{+0.32}$ & $0.56_{-0.08}^{+0.10}$ & $5.61_{-017}^{+0.20}$ \\
\hline FV Tau/c A & $\begin{array}{l}-0.06 \\
0.54_{-0.07}^{+0.05}\end{array}$ & $6.08_{-0.18}^{+0.18}$ & $\begin{array}{l}-0.15 \\
0.55_{-0.07}^{+0.07}\end{array}$ & $6.08_{-0.19}^{+0.18}$ & $0.25_{-0.04}^{+0.08}$ & $\begin{array}{l}-0.17 \\
5.72_{-0.59}^{+0.16}\end{array}$ \\
\hline FV Tau/c B & $\begin{array}{l}-0.07 \\
0.30_{-0.05}^{+0.07}\end{array}$ & $6.67_{-0.20}^{-0.18}$ & $\begin{array}{l}-0.07 \\
0.30_{-0.05}^{+0.07}\end{array}$ & $6.67_{-0.20}^{-0.19}$ & $0.23_{-0.03}^{+0.04}$ & $\begin{array}{r}-0.59 \\
6.48_{-0.19}^{+0.22}\end{array}$ \\
\hline DF Tau A & $0.61_{-0.01}^{+0.05}$ & $5.14_{-0.24}^{-0.20}$ & $0.86_{-0.10}^{-0.05}$ & $\begin{array}{l}5.37_{-0.16}^{+0.120} \\
\end{array}$ & $0.19_{-0.02}^{+0.03}$ & $4.22_{-0.24}^{-0.19}$ \\
\hline DF Tau B & $0.65_{-0.08}^{+0.05}$ & $5.74_{-0.27}^{+0.15}$ & $0.65_{-0.08}^{+0.08}$ & $\begin{array}{l}5.74_{-0.20}^{+0.100} \\
\text { +0. }\end{array}$ & $0.22_{-0.02}^{+0.03}$ & $4.83_{-0.24}^{+0.28}$ \\
\hline 2M04284263 A & $0.16_{-0.04}^{+0.04}$ & $6.50_{-0.19}^{+0.16}$ & $0.16_{-0.04}^{+0.04}$ & $\begin{array}{l}6.50_{-0.19}^{+0.16} \\
\end{array}$ & $0.17_{-0.02}^{+0.02}$ & $6.52_{-0.17}^{+0.24}$ \\
\hline 2M04284263 B & $0.098_{-0.019}^{+0.04}$ & $6.62_{-0.23}^{+0.34}$ & $0.098_{-0.019}^{+0.045}$ & $6.62_{-0.23}^{+0.34}$ & $0.148_{-0.024}^{+0.025}$ & $6.91_{-0.18}^{+0.19}$ \\
\hline UX Tau A & $1.3^{+0.3}{ }^{-0.019}$ & $\begin{array}{l}6.0 .23 \\
6.10^{+0.26}\end{array}$ & $1.9^{+0.3}$ & $\begin{array}{l}-0.23 \\
6.79^{+0.19}\end{array}$ & $1.3^{-0.3} .024$ & $\begin{array}{l}-0.18 \\
6.10^{+0.26}\end{array}$ \\
\hline UX Tau C & $0.16_{-0.04}^{-0.4}$ & $\begin{array}{r}-0.30 \\
6.51_{-0.19}^{+0.16}\end{array}$ & $\begin{array}{c}-0.3 \\
0.16_{-0.04}^{+0.04}\end{array}$ & $\begin{array}{c}{ }^{-0.42} \\
6.51_{-0.19}^{+0.16}\end{array}$ & $0.17_{-0.02}^{-0.4}$ & $\begin{array}{l}6.54_{-0.17}^{+0.20} \\
0.30\end{array}$ \\
\hline FX Tau A & $0.62_{-0.03}^{-0.05}$ & $5.91_{-0.19}^{+0.19}$ & $0.82_{-0.15}^{+0.04}$ & $6.14_{-0.27}^{+0.29}$ & $\begin{array}{l}-0.02 \\
0.30_{-0.06}^{+0.07}\end{array}$ & $\begin{array}{l}-0.17 \\
5.54_{-0.59}^{+0.23}\end{array}$ \\
\hline FX Tau B & $\begin{array}{c}-0.03 \\
0.28_{-0.32}^{+0.16}\end{array}$ & $5.90_{-1.04}^{+0.19}$ & $0.28_{-0.32}^{+0.15}$ & $5.90_{-1.04}^{+0.33}$ & $\begin{array}{l}{ }_{-0.06}^{-0.06} \\
0.18_{-0.04}^{+0 .}\end{array}$ & $5.41_{-0.38}^{+0.56}$ \\
\hline DK Tau A & $0.71_{-0.06}^{+0.09}$ & $5.34_{-0.36}^{+0.04}$ & $1.30_{-0.22}^{+0.16}$ & $5.81_{-0.36}^{+0.34}$ & $0.37_{-0.12}^{-0.13}$ & $4.76_{-0.51}^{+0.65}$ \\
\hline DK Tau B & $\begin{array}{c}{ }^{-0.00} \\
0.61_{-0.04}^{+0.06}\end{array}$ & $\begin{array}{c}6.17_{-0.20}^{+0.30} \\
\end{array}$ & $0.78_{-0.17}^{+0.18}$ & $\begin{array}{c}-0.30 \\
6.38_{-0.27}^{+0.29}\end{array}$ & $0.35_{-0.07}^{+0.08}$ & $5.81_{-0.23}^{+0.21}$ \\
\hline V927 Tau A & $0.42_{-0.06}^{+0.04}$ & $6.21_{-0.18}^{+0.19}$ & $0.42_{-0.06}^{+0.07}$ & $6.21_{-0.18}^{+0.19}$ & $0.24_{-0.04}^{+0.04}$ & $5.93_{-0.31}^{+0.153}$ \\
\hline V927 Tau B & $0.33_{-0.05}^{+0.06}$ & $6.32_{-0.18}^{+0.18}$ & $0.33_{-0.05}^{+0.06}$ & $6.32_{-0.18}^{+0.18}$ & $0.22_{-0.03}^{-0.04}$ & $6.12_{-0.15}^{-0.31}$ \\
\hline HL Tau & $0.83_{-0.10}^{-0.05}$ & $5.93_{-0.25}^{+0.18}$ & $\begin{array}{r}-0.05 \\
1.53_{-0.27}^{+0.21}\end{array}$ & $\begin{array}{r}-0.18 \\
6.51_{-0.35}^{+0.35}\end{array}$ & $\begin{array}{l}-0.03 \\
0.57_{-0.15}^{+0.21}\end{array}$ & $\begin{array}{l}-0.15 \\
5.68_{-0.22}^{+0.33}\end{array}$ \\
\hline XZ Tau A & $0.59_{-0.04}^{+0.07}$ & $5.86_{-0.23}^{+0.20}$ & $0.67_{-0.12}^{-0.14}$ & $5.97_{-0.28}^{-0.35}$ & $0.25_{-0.05}^{+0.06}$ & $5.16_{-0.36}^{+0.52}$ \\
\hline XZ Tau B & $0.46_{-0.17}^{+0.17}$ & $5.36_{-0.36}^{+0.24}$ & $0.46_{-0.30}^{+0.17}$ & $5.36_{-0.36}^{+0.24}$ & $0.17_{-0.02}^{+0.02}$ & $\begin{array}{l}4.44_{-0.25}^{+0.30} \\
\end{array}$ \\
\hline HK Tau A & $0.62_{-0.02}^{+0.03}$ & $6.01_{-0.17}^{-0.36}$ & $\begin{array}{r}-0.30 \\
0.80_{-0.09}^{+0.10}\end{array}$ & $6.24_{-0.21}^{-0.36}$ & $0.32_{-0.04}^{+0.05}$ & $5.69_{-0.45}^{+0.15}$ \\
\hline HK Tau B & $\ldots$ & $>7.7$ & $\ldots$ & $>7.7$ & $\ldots$ & $>7.7$ \\
\hline V710 Tau A & $0.62_{-0.07}^{+0.09}$ & $6.57_{-0.21}^{+0.24}$ & $0.73_{-0.18}^{+0.15}$ & $6.74_{-0.32}^{+0.26}$ & $0.43_{-0.10}^{+0.12}$ & $6.21_{-0.25}^{+0.31}$ \\
\hline V710 Tau B & $0.40_{-0.12}^{+0.14}$ & $6.29_{-0.30}^{+0.32}$ & $0.40_{-0.12}^{+0.18}$ & $6.29_{-0.30}^{+0.32}$ & $0.24_{-0.06}^{+0.10}$ & $6.01_{-0.35}^{+0.22}$ \\
\hline V710 Tau C & $0.35_{-0.07}^{+0.07}$ & $7.24_{-0.25}^{+0.21}$ & $0.35_{-0.07}^{+0.07}$ & $7.24_{-0.25}^{+0.21}$ & $\begin{array}{l}0.29_{-0.04}^{+0.05} \\
\end{array}$ & $7.06_{-0.24}^{+0.24}$ \\
\hline GG Tau Aa & $\begin{array}{l}-0.07 \\
0.73_{-0.08}^{+0.09}\end{array}$ & $5.88_{-0.30}^{+0.25}$ & $1.21_{-0.22}^{-0.07}$ & $6.34_{-0.33}^{+0.25}$ & $0.43_{-0.12}^{+0.04}$ & $5.62_{-0.57}^{-0.24}$ \\
\hline GG Tau Ab & $0.64_{-0.03}^{+0.08}$ & $5.93_{-0.18}^{+0.30}$ & $0.91_{-0.10}^{+0.22}$ & $6.23_{-0.22}^{+0.30}$ & $0.33_{-0.04}^{+0.12}$ & $\begin{array}{c}-0.57 \\
5.59_{-0.46}^{+0.18}\end{array}$ \\
\hline GG Tau Ba & $\begin{array}{c}-0.03 \\
0.104_{-0.020}^{+0.024}\end{array}$ & $5.80_{-0.51}^{+0.18}$ & $\begin{array}{c}-0.10 \\
0.104_{-0.020}^{+0.024}\end{array}$ & $5.80_{-0.51}^{+0.22}$ & $\begin{array}{c}0.3 J_{-0.04}^{-0.014} \\
0.134^{+0.014}\end{array}$ & $\begin{array}{l}5.09-0.46 \\
6.11^{+0.16}\end{array}$ \\
\hline GG Tau Bb & $0.044_{-0.013}^{+0.011}$ & $\begin{array}{r}-0.51 \\
5.63_{-208}^{+0.61}\end{array}$ & $0.044_{-0.013}^{+0.011}$ & $\begin{array}{l}-0.51 \\
5.63_{-208}^{+0.61}\end{array}$ & $0.062_{-0.018}^{+0.021}$ & $\begin{array}{c}-0.44 \\
6.56_{-0.74}^{+0.14}\end{array}$ \\
\hline UZ Tau Aa & $0.62_{-0.03}^{+0.05}$ & $5.83_{-020}^{+0.19}$ & $0.84_{-0.14}^{+0.18}$ & $6.06_{-0.27}^{+0.08}$ & $0.29_{-0.05}^{+0.07}$ & $5.27_{-0.42}^{+0.43}$ \\
\hline UZ Tau Ab & $0.26_{-0.35}^{+0.20}$ & $5.76_{-120}^{+0.38}$ & $0.26_{-0.35}^{-0.14}$ & $5.76_{-120}^{+0.38}$ & $0.17_{-0.04}^{+0.06}$ & $5.20_{-0.34}^{+0.67}$ \\
\hline UZ Tau Ba & $\begin{array}{l}-0.35 \\
0.59_{-0.01}^{+0.05}\end{array}$ & $\begin{array}{r}-1.20 \\
5.81_{-0.19}^{+0.19}\end{array}$ & $\begin{array}{l}-0.35 \\
0.68_{-0.08}^{+0.07}\end{array}$ & $\begin{array}{r}-1.20 \\
5.93_{-0.17}^{+0.19}\end{array}$ & $\begin{array}{c}-0.04 \\
0.25_{-0.03}^{+0.04}\end{array}$ & $\begin{array}{l}5.09_{-0.38}^{+0.34} \\
\end{array}$ \\
\hline UZ Tau Bb & $0.49_{-0.09}^{+0.07}$ & $5.98_{-0.20}^{+0.18}$ & $0.49_{-0.09}^{+0.08}$ & $5.98_{-0.20}^{+0.18}$ & $0.22_{-0.03}^{-0.03}$ & $5.33_{-0.31}^{+0.53}$ \\
\hline GH Tau A & $\begin{array}{r}-0.09 \\
0.58_{-0.05}^{+0.02}\end{array}$ & $6.15_{-0.20}^{-0.19}$ & $\begin{array}{r}-0.09 \\
0.61_{-0.07}^{+0.09}\end{array}$ & $\begin{array}{r}-0.20 \\
6.20_{-0.18}^{+0.20}\end{array}$ & $\begin{array}{l}0.0 .03 \\
0.29_{-0.04}^{+0.05}\end{array}$ & $\begin{array}{l}-0.31 \\
5.77_{-0.47}^{+0.15}\end{array}$ \\
\hline GH Tau B & $\begin{array}{l}-0.05 \\
0.58_{-0.02}^{+0.02}\end{array}$ & $5.98_{-0.20}^{+0.20}$ & $\begin{array}{l}-0.07 \\
0.64_{-0.07}^{+0.08}\end{array}$ & $6.07_{-0.18}^{+0.18}$ & $\begin{array}{c}-0.04 \\
0.27_{-0.03}^{+0.04}\end{array}$ & $\begin{array}{r}-0.47 \\
5.50_{-0.49}^{+0.29}\end{array}$ \\
\hline IS Tau A & $0.67^{+0.04}$ & $6.11_{-020}^{+0.21}$ & $0.99_{-0.09}^{+0.09}$ & $6.46_{-0.19}^{+0.19}$ & $0.39^{+0.06}$ & $\begin{array}{r}5.77_{-018}^{+0.19}\end{array}$ \\
\hline IS Tau B & $0.30_{-0.06}^{+0.06}$ & $6.62_{-0.20}^{+0.20}$ & $0.30_{-0.05}^{+0.06}$ & $6.62_{-0.20}^{+0.21}$ & $0.23_{-0.03}^{+0.05}$ & $6.42_{-0.18}^{+0.21}$ \\
\hline HN Tau A & $0.85_{-0.10}^{-0.05}$ & $6.27_{-0.25}^{+0.20}$ & $1.35_{-0.16}^{-0.05}$ & $6.85_{-0.34}^{+0.20}$ & $0.65_{-0.18}^{+0.03}$ & $6.05_{-0.32}^{+0.38}$ \\
\hline HN Tau B & $0.20_{-0.10}^{+0.10}$ & $\begin{array}{l}-0.25 \\
6.59_{-0.49}^{+0.45}\end{array}$ & $0.20_{-0.10}^{+0.16}$ & $\begin{array}{l}6.59_{-0.49}^{+0.34} \\
\end{array}$ & $0.19_{-0.05}^{+0.18}$ & $6.54_{-0.23}^{+0.32}$ \\
\hline IT Tau A & $1.0_{-01}^{+0.10}$ & $5.96_{-0.21}^{+0.49}$ & $\begin{array}{l}-0.10 \\
1.8_{-0.2}^{+0.2}\end{array}$ & $\begin{array}{r}-0.49 \\
6.76_{-0.39}^{+0.18}\end{array}$ & $\begin{array}{c}-0.05 \\
1.0_{-0.3}^{+0.3}\end{array}$ & $5.96_{-0.32}^{+0.33}$ \\
\hline IT Tau B & $0.28_{-032}^{+0.15}$ & $5.90_{-104}^{+0.33}$ & $0.28_{-0.12}^{+0.15}$ & $5.90_{-104}^{+0.33}$ & $0.18_{-0.04}^{+0.06}$ & $5.42_{-0.58}^{+0.55}$ \\
\hline Haro 6-28 A & $0.52^{-0.077}$ & $6.80_{-0.22}^{+0.21}$ & $0.52_{-0.08}^{+0.072}$ & $6.80_{-0.22}^{+0.04}$ & $0.35_{-0.06}^{+0.04}$ & $6.45^{+0.24}$ \\
\hline Haro 6-28 B & $\begin{array}{l}-0.08 \\
0.30_{-0.05}^{+0.06}\end{array}$ & $6.60_{-0.20}^{+0.22}$ & $0.30_{-0.05}^{+0.08}$ & $6.60_{-0.20}^{+0.22}$ & $0.23_{-0.03}^{+0.06}$ & $6.39_{-0.17}^{+0.21}$ \\
\hline $2 \mathrm{M} 04414565$ & $\begin{array}{l}-0.05 \\
0.37_{-0.05}^{+0.08}\end{array}$ & $6.54_{-0.20}^{+0.20}$ & $\begin{array}{l}-0.05 \\
0.37_{-0.05}^{+0.08}\end{array}$ & $6.54_{-0.20}^{-0.20}$ & $0.25_{-0.03}^{-0.03}$ & $6.26_{-0.16}^{+0.21}$ \\
\hline 2M04414489 & $\begin{array}{l}-0.05 \\
0.027_{-0.009}^{+0.006}\end{array}$ & $6.47_{-0.94}^{-0.44}$ & $\begin{array}{l}-0.05 \\
0.027_{-0.009}^{+0.006}\end{array}$ & $6.47_{-0.94}^{-0.20}$ & $0.022_{-0.006}^{+0.003}$ & $6_{-0.47}^{+0.02}$ \\
\hline
\end{tabular}


Table 2

(Continued)

\begin{tabular}{|c|c|c|c|c|c|c|c|}
\hline Name & $M\left(M_{\odot}\right)$ & $\log (\tau)(\mathrm{yr})$ & \multicolumn{2}{|c|}{ (Lyon) } & \multicolumn{3}{|c|}{ (DM97) } \\
\hline LkHa332-G2 A & $0.64_{-0.02}^{+0.03}$ & $5.90_{-0.18}^{+0.19}$ & $0.92_{-0.10}^{+0.10}$ & $6.20_{-0.21}^{+0.20}$ & $0.33_{-0.04}^{+0.04}$ & & $5.56_{-0.50}^{+0.19}$ \\
\hline LkHa332-G2 B & $0.52_{-0.07}^{+0.05}$ & $6.15_{-0.18}^{+0.18}$ & $0.52_{-0.07}^{+0.07}$ & $6.15_{-0.19}^{+0.19}$ & $0.26_{-0.04}^{+0.05}$ & & $\begin{array}{l}5.81_{-0.53}^{+0.140} \\
0\end{array}$ \\
\hline V955 Tau A & $0.72_{-0.04}^{+0.05}$ & $5.73_{-0.24}^{+0.22}$ & $1.24_{-0.10}^{+0.11}$ & $6.20_{-0.22}^{+0.22}$ & $0.41_{-0.06}^{+0.04}$ & & $5.51_{-0.55}^{+0.15}$ \\
\hline V955 Tau B & $0.45_{-0.07}^{+0.08}$ & $6.46_{-0.20}^{+0.23}$ & $0.45_{-0.07}^{+0.08}$ & $6.46_{-0.20}^{+0.23}$ & $0.28_{-0.04}^{+0.06}$ & & $6.12_{-0.16}^{+0.20}$ \\
\hline UY Aur A & $0.66_{-0.02}^{+0.03}$ & $5.56_{-0.20}^{+0.20}$ & $\begin{array}{l}1.07_{-0.12}^{+0.12} \\
\end{array}$ & $5.95_{-0.25}^{+0.23}$ & $0.31_{-0.04}^{+0.04}$ & & $4.94_{-0.31}^{+0.56}$ \\
\hline UY Aur B & $0.62_{-0.08}^{+0.02}$ & $5.84_{-0.23}^{+0.15}$ & $0.62_{-0.07}^{+0.08}$ & $5.84_{-0.20}^{-0.25}$ & 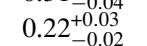 & & $4.99_{-0.24}^{+0.43}$ \\
\hline 2M04554757 & $0.20_{-0.03}^{+0.03}$ & $6.27_{-0.17}^{+0.17}$ & $0.20_{-0.03}^{+0.03}$ & 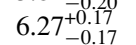 & $\begin{array}{l}-0.02 \\
0.17_{-0.01}^{+0.02}\end{array}$ & & $\begin{array}{c}-0.24 \\
6.22_{-0.13}^{+0.14}\end{array}$ \\
\hline $2 \mathrm{M} 04554801$ & $\begin{array}{c}-0.03 \\
0.092_{-0.012}^{+0.008}\end{array}$ & $\begin{array}{r}-0.17 \\
6.96_{-0.27}^{+0.76}\end{array}$ & $\begin{array}{c}0.092_{-0.012}^{+0.03} \\
0.008\end{array}$ & $6.96_{-0.27}^{+0.17}$ & $0.133_{-0.016}^{+0.015}$ & & $\begin{array}{l}-0.13 \\
7.13_{-0.15}^{+0.16}\end{array}$ \\
\hline RW Aur A & $1.4_{-0.7}^{+0.6}$ & $5.85_{-0.53}^{+0.44}$ & $2.1_{-0.5}^{+0.3}$ & $6.32_{-0.42}^{-0.54}$ & $1.4_{-0.7}^{+0.6}$ & & $5.85_{-0.53}^{+0.44}$ \\
\hline RW Aur B & $0.86_{-0.10}^{+0.11}$ & $\begin{array}{l}6.40_{-0.26}^{+0.26} \\
x_{-0.25}\end{array}$ & $1.26_{-0.14}^{+0.14}$ & $6.96_{-0.33}^{+0.29}$ & $0.69_{-0.19}^{+0.24}$ & & $6.19_{-0.35}^{+0.39}$ \\
\hline
\end{tabular}

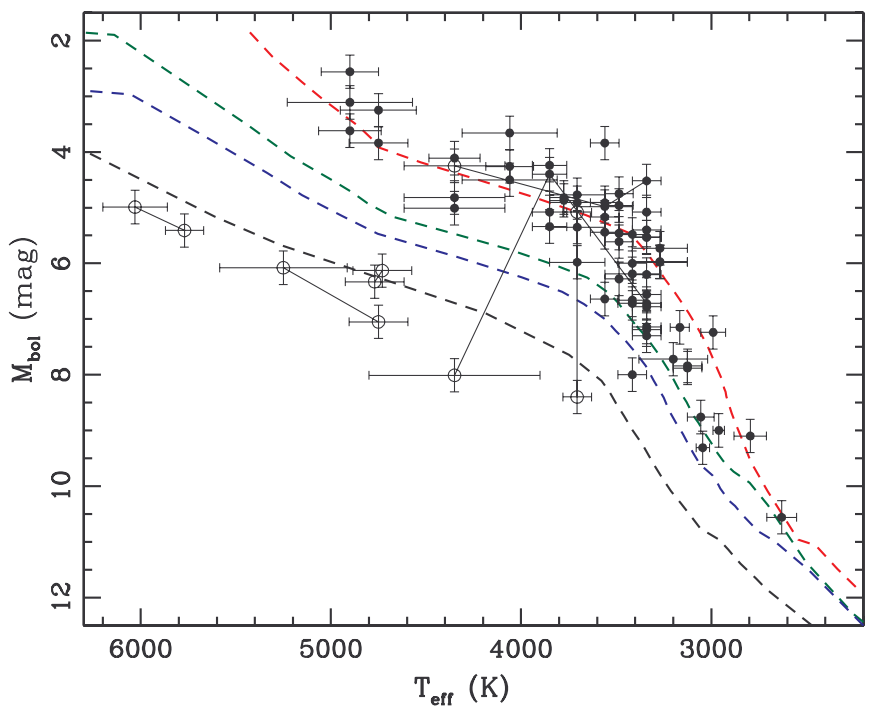

Figure 1. HR diagram for all components of all sample binaries. The binary components that we rejected due to known systematic errors (Section 4) are shown with open circles, while the rest of our sample is shown with filled circles. The dashed lines denote isochrones at $1 \mathrm{Myr}$ (red), $5 \mathrm{Myr}$ (green), $10 \mathrm{Myr}$ (blue), and $50 \mathrm{Myr}$ (black). Most Taurus members fall along the $1 \mathrm{Myr}$ isochrone, but 10 fall below the $5 \mathrm{Myr}$ isochrone. Three binary pairs that fall mutually below this limit might be associated with the more distant Perseus star-forming complex, while individual components that are associated with apparently young binary companions could be seen in scattered light or have incorrect spectral types. We use solid lines to connect each binary pair with one or more rejected components.

(A color version of this figure is available in the online journal.)

Most of the binary components below this isochrone are known to be anomalous.

The warmest anomalous members (HBC 352-357) have been classified as Taurus members for several decades (e.g., Walter et al. 1988; Herbig \& Bell 1988; Kenyon \& Hartmann 1995) based on their X-ray emission and (for HBC 352-355) their radial velocities. However, these stars are located at the far western edge of Taurus, well away from the central cloud cores, and their underluminosity has been recognized since their discovery. Few membership surveys have extended so far in this direction from the clouds, so it is unknown whether these stars are surrounded by a more extensive coeval population. Given their proximity in projection to the Perseus complex, it seems plausible that they are associated with that more distant, but similarly young population. Since both members of each binary pair seem to be equally anomalous, this seems to be a reasonable explanation for their low positions on the HR diagram; we therefore choose to remove them from our sample for all subsequent analysis.

Three of the other binary companions are known sources seen only in scattered light due to the presence of an edge-on circumstellar disk. Stapelfeldt et al. (1998) used HST and AO observations to show that the optical and NIR flux from HK Tau B comes from extended nebulosity, with no recognizable flux coming directly from the central star. Krist et al. $(1995,1998)$ found similar results from HST imaging of HL Tau and Haro 6-5B, respectively. HK Tau and Haro 6-5B appear significantly underluminous in our HR diagram since this reflected light only represents a small fraction of each star's total emitted flux. Surprisingly, HL Tau does not appear underluminous, which suggests that its luminosity might be dominated by scattered light in the optical and direct flux from the central star in the $J$ band. However, we have chosen conservatively to omit all three companions from our analysis of relative binary ages.

The binary component Haro 6-28 A sits just below the $5 \mathrm{Myr}$ isochrone, so a further analysis of its scattered-light properties might be worthwhile in the future. However, its companion Haro 6-28 B sits just above the $5 \mathrm{Myr}$ isochrone, so the inferred ages of the two components are mutually consistent. Barring a systematic uncertainty for the binary system, this consistency suggests that Haro 6-28 AB is genuinely one of the oldest systems in Taurus. Haro 6-28 was originally identified as an H $\alpha$ emission line star (Haro et al. 1953; Cohen \& Kuhi 1979) and at least one component has a $1.3 \mathrm{~mm}$ excess indicative of a disk, so its youth seems secure. ${ }^{1}$

Finally, two companions (V710 Tau C and 2M04554801) sit below the $5 \mathrm{Myr}$ isochrone without any obvious explanation. Their optical spectra appear to be accurately classified (Luhman 2004; Kraus \& Hillenbrand 2009), so a large error in temperature seems unlikely. They are associated with stars that appear youthful, so membership in a different population also does not explain their anomalously old apparent ages. V710 Tau C has not been observed at high spatial resolution, but it shows a very significant $K$ band excess in 2MASS $(J-K \sim 2.2)$, so it might possess an edge-on circumstellar disk. 2M04554801 appears to be a point source in $K$ band imaging with Keck LGSAO (A. Kraus \& L. Hillenbrand 2009, in preparation), so if the star is seen in scattered light in the near-infrared, then the scattering region must be smaller than for other sources with edge-on disks. The original discovery spectrum shows obvious signatures of youth (K. Luhman 2009, private communication),

\footnotetext{
1 As demonstrated by Carpenter et al. (2006) for Upper Sco, massive circumstellar disks are relatively rare $(f \sim 5 \%)$ for $\sim 0.3-0.5 M_{\odot}$ stars by the age of $\sim 5 \mathrm{Myr}$, though not as rare as for higher mass stars.
} 


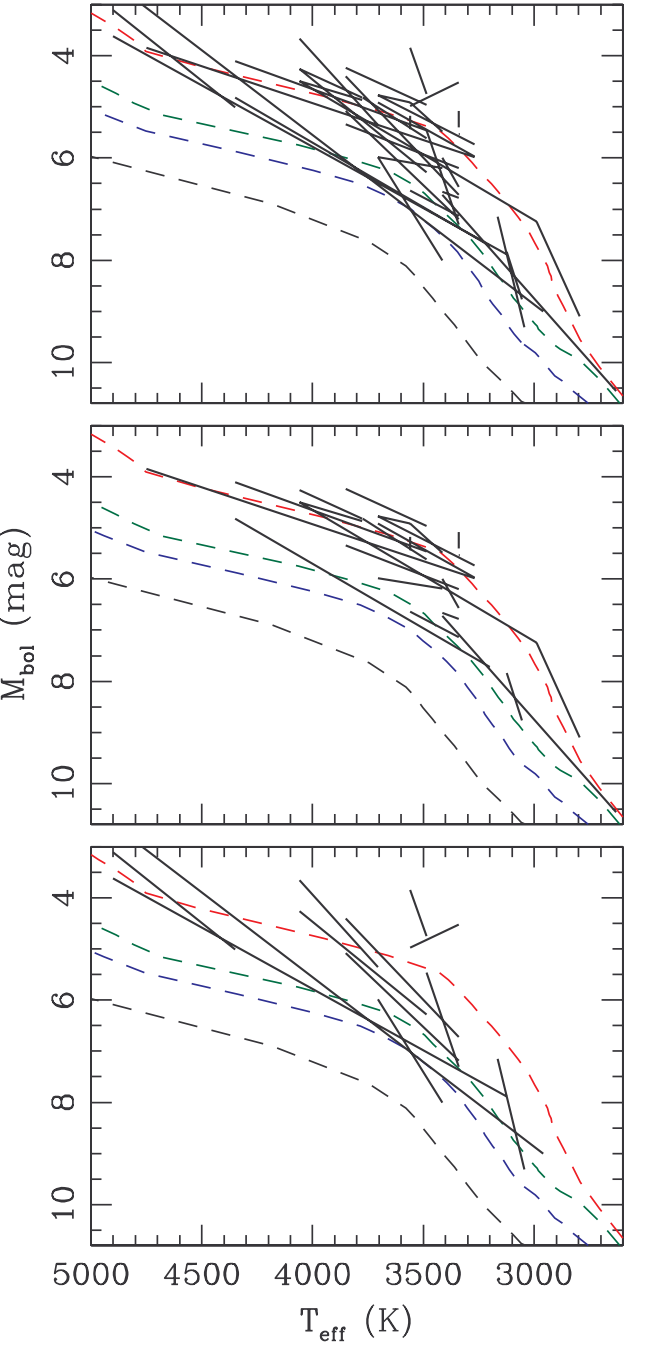

Figure 2. HR diagram for the binary pairs in our sample, where each pair is connected by a line. The top panel shows all systems, whereas following the text in Section 5.1, the other panels show only systems with $|\Delta \log \tau|<0.4$ dex (middle) and $|\Delta \log \tau|>0.4$ dex (bottom). The binary systems in our sample trace the approximate contours of stellar evolutionary models, suggesting that the overall trend is correct, but our detailed results are more easily described in terms of the inferred stellar ages (Figures 3-6).

(A color version of this figure is available in the online journal.)

and the optical/NIR spectral energy distribution (SED) does not show any of the characteristic signs of an edge-on disk, so the explanation for its underluminosity is currently unknown. We cannot justify removing either star from our sample, but both systems should be regarded with appropriate skepticism.

In the following analysis, we will omit all of the $\mathrm{HBC}$ sources that have questionable membership. We will retain Haro 6-5B and HL Tau for a more in-depth study of high-order multiple systems, but will omit all three confirmed scattered light systems for testing coevality. We will retain Haro 6-28 A, V710 Tau C, and $2 \mathrm{M} 04554801$ since there is no conclusive evidence to suggest that they suffer from a systematic bias.

\section{THE COEVALITY OF YOUNG BINARY SYSTEMS}

\subsection{The Relative Ages of Binary Systems}

The first step of our analysis is to determine whether binary systems appear more coeval than the association as a whole. The timescale for star formation across an entire region could
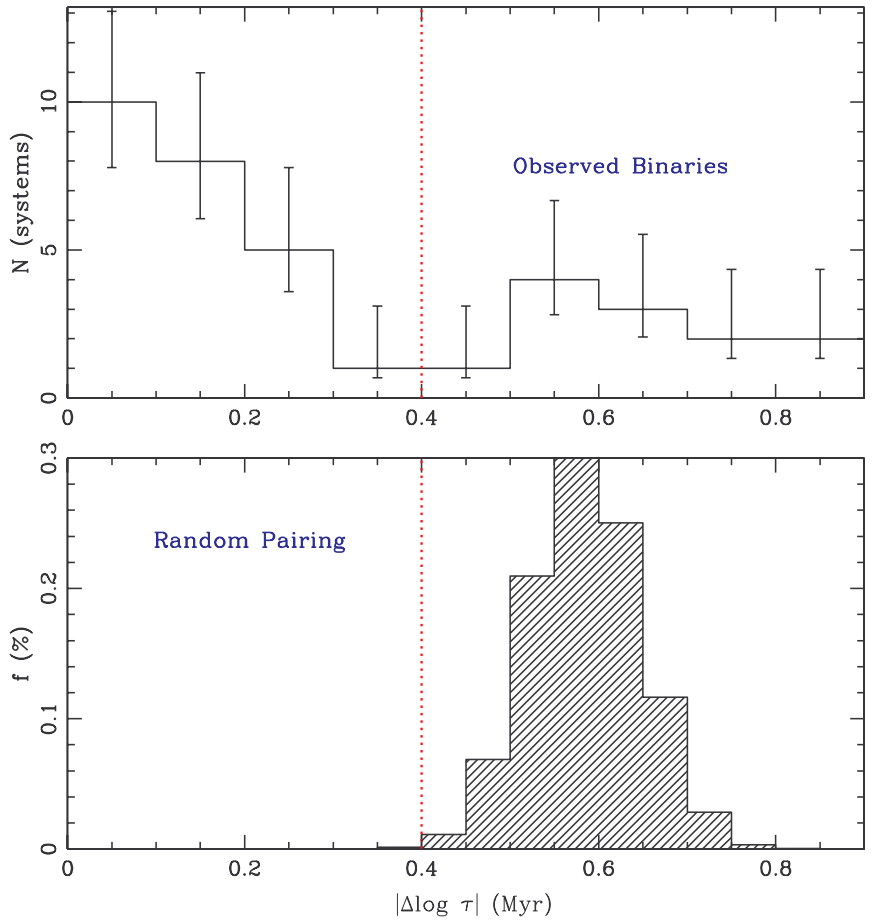

Figure 3. Top: distribution of differences in logarithmic age, $|\Delta \log \tau|$, for all 36 pairs of stars in our sample. The rms scatter in $|\Delta \log \tau|, \sigma=0.40 \mathrm{dex}$, is indicated by a red dotted line. Bottom: distribution of rms scatter for a set of 10,000 simulated binary populations that were constructed by randomly pairing primaries with secondaries. As before, we show the rms scatter of our observed population with a red dotted line; only 14 of the 10,000 simulated populations have $\sigma \lesssim 0.40 \mathrm{dex}$, indicating that our binary pairs are more coeval than Taurus at $\sim 3 \sigma$ significance.

(A color version of this figure is available in the online journal.)

be as long as 10 Myr (Mouschovias 1976; Shu 1977), while the formation of a single star system should proceed on the dynamical timescale of $\sim 0.1-0.2 \mathrm{Myr}$ (Shu et al. 1987). An upper limit on the noncoevality of binary systems will provide a direct constraint on the formation timescale for binary systems with respect to the entire association.

In Figure 2 (top), we show the same HR diagram as in Figure 1, minus the objects we eliminated in the discussion above, where each of the binary pairs is connected by a line. The overall trend for binary pairs is to define lines that roughly parallel the theoretical isochrones, which is expected since the models have been chosen by comparison to the Taurus singlestar sequence. However, as we determine quantitatively below, some pairs fall on significantly different isochrones. This type of plot provides a summary of the underlying data, but it is hard to draw any firm conclusions regarding the overall coevality or possible dependence of coevality on binary parameters. A more detailed statistical analysis must be pursued using the inferred stellar ages, as has been concluded by past studies of binary ages (e.g., Hartigan et al. 1994; White \& Ghez 2001; Hartigan $\&$ Kenyon 2003).

In Figure 3 (top), we show a histogram of the absolute difference in the inferred logarithmic age, $|\Delta \log \tau|=\mid \log \tau_{\text {prim }}-$ $\log \tau_{\mathrm{sec}}$, for each of our binary pairs. The root mean square (rms) scatter in $|\Delta \log \tau|$ among our sample is 0.40 dex and should encompass all observational uncertainties as well as any intrinsic age spread for binary pairs. Most of the model-predicted masses fall in the range $0.3 M_{\odot}<M<0.9 M_{\odot}$, with some masses extending as high as $1.5 M_{\odot}$ and as low as $0.03 M_{\odot}$. We have 
compared the scatter in binary component ages with that of the overall Taurus population by using a bootstrap Monte Carlo routine to simulate 10,000 populations where we pair each primary with another randomly selected secondary. In Figure 3 (bottom), we show the distribution of all rms scatter measurements for these simulated pairs; only 14 realizations of our simulation $(\sim 0.15 \%)$ have an rms scatter of 0.40 dex or less, indicating that binaries are more coeval than the overall Taurus population at a significance of $\sim 3 \sigma$. The typical age differences for binary systems and random pairs are similar to those measured by White $\&$ Ghez (2001); they used a similar sample, but estimated effective temperatures using dereddened $V-I$ photometry instead of spectral types.

As described in Section 3, the observational uncertainties for our sample allow us to estimate the corresponding uncertainty in each binary component's age, and thus in the degree of coevality. These estimated uncertainties vary significantly across our sample, but the median, the mean, and the quadratic mean of these uncertainties $(0.33,0.42$, and 0.48 dex in measured $|\Delta \log \tau|$, respectively) are all similar to the standard deviation for our sample. This strongly suggests that much of the total error budget is dominated by observational errors, and therefore that model-related errors and the true dispersion in relative ages for binary components are both $\ll 0.40$ dex.

However, we must also consider whether a single distribution is adequate for describing all binary systems, as there are several effects that could bias one binary component's age by a significant amount (including overluminosity due to unresolved additional multiplicity or underluminosity because an object is seen in scattered light). We could expect a narrow distribution centered close to zero and broadened by the observational errors (corresponding to unaffected binary systems) plus a secondary peak away from zero (for systems affected by unusual phenomena such as those mentioned above). The observed distribution seems to match this expectation, with most systems concentrated at $|\Delta \log \tau|<0.3$ dex and an extended tail at $|\Delta \log \tau| \gtrsim 0.5$ dex. If we omit the extended tail and compute the standard deviation of the binary age dispersion for only systems with $|\Delta \log \tau|<0.4$ dex, we find a dispersion of $\sigma_{|\Delta \log \tau|} \sim 0.16 \mathrm{dex}$, corresponding to a typical factor of 1.5 in relative age. This dispersion is actually lower than the typical uncertainties we estimated above for $|\Delta \log \tau|$, which suggests that we might have been too conservative in estimating observational uncertainties.

The extended tail includes one sample member that we suggested to be a candidate edge-on disk (V710 Tau C) based on its underluminosity and extremely red $J-K$ color. The tail also includes two possible hierarchical triple systems, DK Tau and XZ Tau, which were suggested to be possible hierarchical triples by Jensen et al. (2004) and Carrasco-Gonzalez et al. (2009). Polarization measurements by Jensen et al. (2004) indicate that the component disks in $\mathrm{DK}$ Tau $\mathrm{AB}$ are misaligned, unlike most other double-disk systems in Taurus. Radio observations by Carrasco-Gonzalez et al. (2009) show that XZ Tau B has a double-peaked distribution that could result from a $\sim 13 \mathrm{AU}$ binary companion.

In light of this possible bimodality, it is worthwhile to return to Figure 2 and plot only the "coeval" sample $(|\Delta \log \tau|<0.4$ dex; center) and the "noncoeval" sample ( $|\Delta \log \tau|>0.4$ dex; right). This division reveals a startling trend; among the "noncoeval" subsample, 11 of the 12 systems have a significantly younger primary star. As we show in Appendix A, the model-derived ages in this mass range do not show a mass-dependent trend, so the tendency for some binary primaries to appear younger must be either a genuine result of the formation process or a result of binary-specific systematic uncertainties. Given the clear discrepancy with respect to the apparently coeval majority of our sample, we strongly suspect that systematic errors are to blame.

The fraction of apparently coeval binary systems in our sample $(24 / 36)$ is identical to the fraction identified by the survey of Hartigan et al. (1994). In a sample of binary systems in Taurus and Orion (which included many Taurus binaries that we have rejected as hierarchical multiples), they found that $17 / 26$ had $\Delta \log (L)<0.24$ dex, corresponding roughly to $|\Delta \log \tau| \lesssim 0.4$ dex. On its face, this result suggests that all of the improvements in evolutionary models, spectral type assessments, and multiplicity surveys in the past 15 years have only served to cut the standard deviation in $|\Delta \log \tau|$ for coeval systems from 0.23 dex to 0.16 dex. However, there is one significant difference. All of our noncoeval systems possess apparently younger primaries, while all of the corresponding systems in the Hartigan et al. sample possess apparently older primaries. There is little overlap between our samples since many wide Taurus binaries have since been discovered to be hierarchical multiples, so one possible explanation is that our stringent multiplicity vetting simply allows another systematic error to dominate. There are three likely culprits for the systematic error that makes some systems appear noncoeval.

First, the noncoevality could result from unrecognized highorder multiplicity. The binary fraction is higher among solartype stars than lower mass stars (Duquennoy \& Mayor 1991; Fischer \& Marcy 1992). If this trend also applies to the fragmentation of binary components into high-order multiples, then we might expect more binary primaries to be unresolved pairs (which would then appear to be a single overluminous star). An overluminosity by $0.75 \mathrm{mag}$ (denoting an equal-mass binary pair) should correspond to an apparent age discrepancy of $\sim 0.5$ dex, which matches the observed trend. However, our sample includes many objects with similar temperatures (and thus presumably similar masses), and as we will show in a future paper (A. Kraus \& L. Hillenbrand 2009, in preparation), the frequency of binaries at separations $\lesssim 50-100 \mathrm{AU}$ in Taurus is nearly constant for all masses $\gtrsim 0.3 M_{\odot}$. We might expect a significant excess of apparently younger primary stars if the secondary masses fell significantly below this limit, but few of our targets do.

The other two possible explanations are tied to the properties of circumstellar disks. Surveys of protoplanetary disks in binary systems have suggested that disks might be more likely to form or persist around the primary than the secondary (e.g., Monin et al. 2007), even though lower mass stars generally retain their disks longer (e.g., Carpenter et al. 2006). We determined most stellar luminosities from the $K$ band flux, so a disk excess could have led to significant overestimation of those luminosities. In addition, the disks of high-mass stars boast more substantial NIR excesses than those of low-mass stars and brown dwarfs (e.g., Meyer et al. 1997 versus Liu et al. 2003), so the magnitude of the luminosity overestimate should also be larger for primaries than that for secondaries.

All of these explanations should be investigated and ruled out before an astrophysical explanation is considered. In particular, systematic effects from disks should be mitigated by estimating stellar luminosities using the least contaminated filter $(J)$ and by modeling the circumstellar dust emission using JHKL photometry so that any remaining excess can be subtracted. However, 


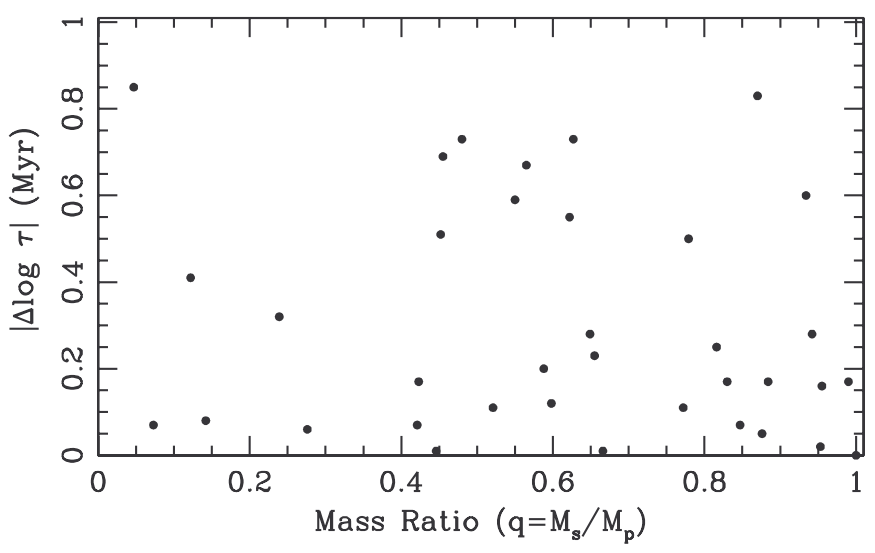

Figure 4. Difference in binary component age, $|\Delta \log \tau|$, as a function of binary mass ratio. We see no evidence of a trend with $q$, as the standard deviations in $|\Delta \log \tau|$ for $q<0.3$ and the full sample are the same for all pairs $(0.41$ dex vs. $0.40 \mathrm{dex})$ and for the apparently coeval subset ( 0.17 vs. $0.16 \mathrm{dex})$.

our preliminary disk census suggests that NIR excess contamination might play only a modest role in biasing relative binary ages (unless the disk directly obscures the central star, making it appear in scattered light; Section 4). Of the 24 pairs that appear coeval, 20 have at least one disk (where $\geqslant 4$ are mixed pairs and $\geqslant 11$ are double-disk systems). This presents little contrast to the 12 apparently noncoeval pairs, of which nine have at least one disk (with three mixed pairs and six double-disk systems). The similar and nontrivial fractions of mixed pairs are difficult to explain if disk biases dominate, though double-disk systems could appear coeval if both binary components' luminosities are biased upward by the same amount.

\subsection{The Role of System Parameters in Binary Coevality}

The detailed physics of multiple star formation are still poorly understood, so any apparent trends in the coevality of binary systems could yield valuable new constraints on theoretical models. One-third of the systems in our sample appear noncoeval, so any such trend could be identified among the 12 noncoeval binary pairs. The three binary properties that we can test against system coevality are the component mass ratio, the total system mass, and the system projected separation. The degree of coevality as a function of separation across the entire association could also constrain the large-scale star formation processes, so we also analyze the coevality as a function of separation between all pairs of stars in our sample.

In Figure 4, we plot the difference in system age $|\Delta \log \tau|$ as a function of binary mass ratio. If binaries truly formed noncoevally, then we might expect the systems with the most extreme or most similar mass ratios to show the largest discrepancy in ages. However, the dispersion in $|\Delta \log \tau|$ for the five systems with $q<0.3$ is $0.41 \mathrm{dex}$, similar to the overall dispersion for our full sample $(0.40 \mathrm{dex})$. If we limit this analysis to only the apparently coeval population $(|\Delta \log \tau|<0.40 \mathrm{dex})$, the dispersions are 0.17 dex and 0.16 dex, respectively. This indicates that there is no strong trend for reduced coevality in these extreme systems. Hartigan et al. (1994) also found no such trend in their sample.

In Figure 5, we plot $|\Delta \log \tau|$ as a function of the total system mass. Most of our sample spans only a limited mass range $\left(0.7-1.5 M_{\odot}\right)$, but we see no evidence of a mass-dependent trend. Our sample includes only four systems with a total mass of $<0.5 M_{\odot}$, but we also see no significant trend for a higher scatter in ages. The dispersion $(0.36 \mathrm{dex})$ is similar to

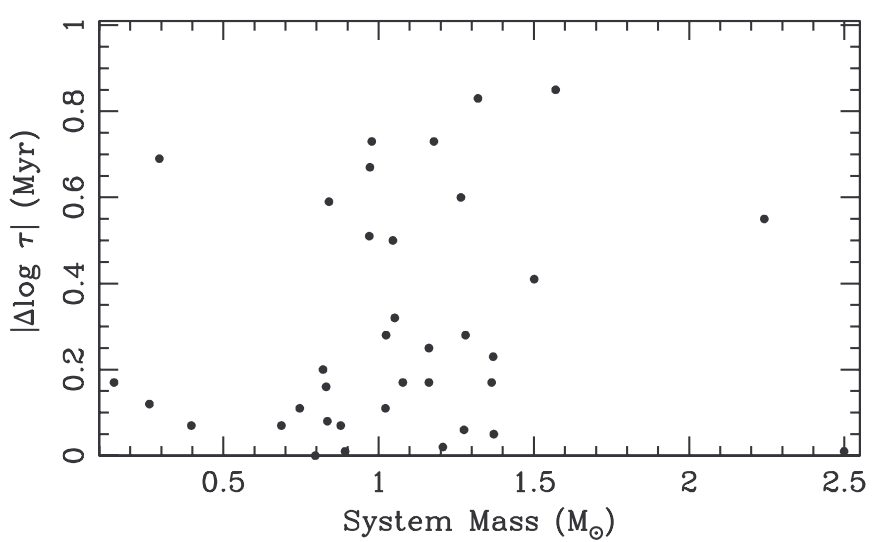

Figure 5. Difference in binary component age, $|\Delta \log \tau|$, as a function of system mass. We see no trend for low-mass systems to appear more discrepant, but are unable to test systems with $M \gtrsim 1.5 M_{\odot}$ and can only test a handful of systems with $M \lesssim 0.7 M_{\odot}$.

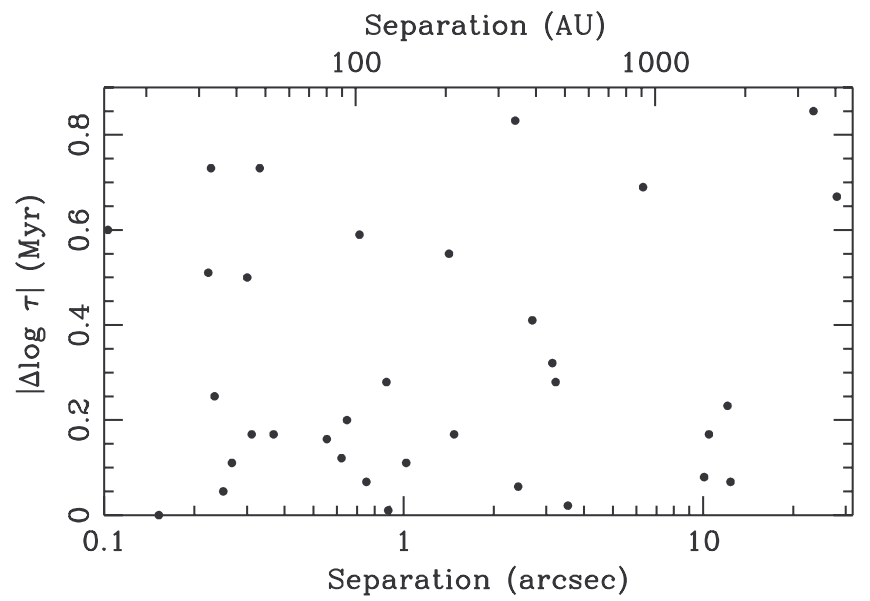

Figure 6. Difference in binary component age, $|\Delta \log \tau|$, as a function of binary separation. The standard deviations in $|\Delta \log \tau|$ for the inner and outer halves are 0.37 dex vs. 0.42 dex (for the full set) and 0.14 dex vs. $0.18 \mathrm{dex}$ (for the coeval subset); in both cases, the inner and outer halves are divided at $800 \mathrm{AU}$. This indicates that binary systems of all separations are similarly coeval.

that of the full sample, though almost entirely dominated by one system (2M04554757+2M04554801). Our sample includes only two high-mass pairs, RW Aur AB and V773 Tau Aab, for which we measure age discrepancies of 0.55 dex and 0.01 dex, respectively.

In Figure 6, we plot $|\Delta \log \tau|$ as a function of system separation. If the separation of a binary system scales with the protostellar core size when fragmentation occurred, then wider systems should typically fragment at an earlier stage than closer systems. This suggests that wide pairs might fragment earlier and evolve more independently, possibly yielding binary components with a larger dispersion in apparent ages. However, as for the previous figures, this comparison does not indicate any significant role of separation in establishing the binary component ages. The inner and outer halves of the sample (divided at $800 \mathrm{AU}$ ) have dispersions of 0.37 dex versus 0.42 dex (for the full set) and 0.14 versus 0.18 dex (for the coeval subset). We conclude that binary systems of all separations are similar coeval to within our observed limits.

\subsection{The Intra-association Coevality of Young Stars}

The lack of a separation-dependent trend in differential age begs an important question. If binary pairs are similarly coeval with their associated components, but significantly more 


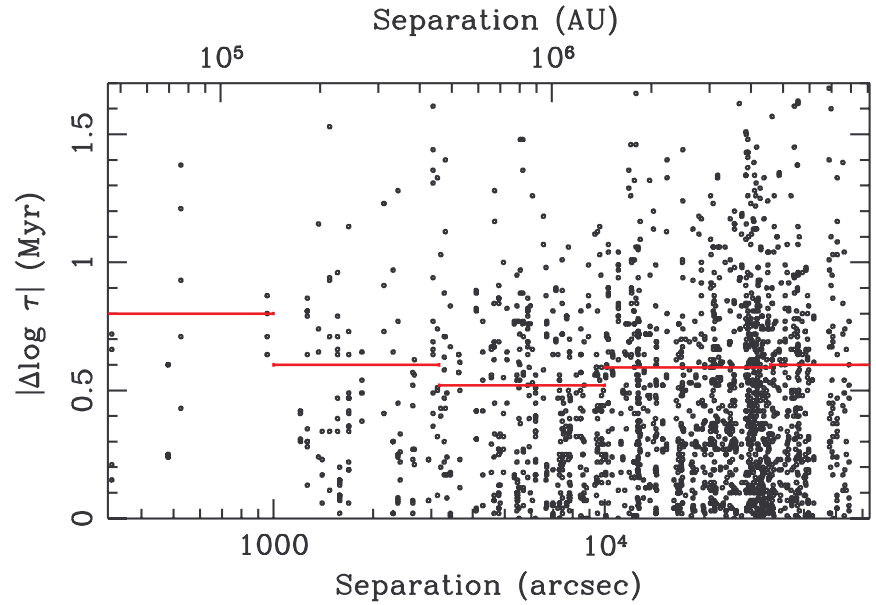

Figure 7. Difference in age as a function of (large-scale) separation for all possible pairs of Taurus members among our binary sample. We also show the dispersion for all pairs in bins 0.5 dex wide (red lines). The sample is insufficient for testing coevality on scales smaller than $\sim 1000^{\prime \prime}(0.7 \mathrm{pc})$, but all pairs on larger spatial scales have a dispersion of $\sim 0.6 \mathrm{dex}$, which is similar to the age dispersion for random pairs of Taurus members ( 0.58 dex; Section 5.1). This result indicates that the coevality seen for binary systems is limited to smaller spatial scales, and perhaps only to binary systems themselves.

(A color version of this figure is available in the online journal.)

coeval than the association as a whole, then what is the form of the transition between these regimes? Are adjacent (but unassociated) Taurus members more coeval than distant members, or is the age spread similar across all spatial scales greater than the binary separation regime? The distribution of Taurus members has been suggested to represent a small number of subclusters (e.g., Gomez et al. 1993) with radii of $\sim 1^{\circ}-2^{\circ}$ $(\sim 5 \mathrm{pc})$, though it is unclear whether those groups are distinct from the large-scale filamentary (and possibly fractal) structure (Kraus \& Hillenbrand 2008). If these apparent groupings are closely associated, then stars separated by $<5$ pc might be more coeval than the wider association.

In Figure 7, we address this question by plotting $|\Delta \log \tau|$ as a function of separation for all possible pairs of the primary and secondary stars in our binary sample. We cannot draw any conclusions for separations of $30^{\prime \prime}-1000^{\prime \prime}$ (5000 AU to $0.7 \mathrm{pc}$ ) due to small number statistics, but the dispersion at larger separations (as indicated by the standard deviation in bins 0.5 dex wide) is consistently $\sim 0.6$ dex across the entire separation range. This result suggests that the coevality we see for binary systems (Figure 6) is limited to scales of $<0.7 \mathrm{pc}$. We cannot determine if this coevality is limited exclusively to binary systems, though; as we showed in our analysis of the spatial distributions of stars (using two-point correlation functions; Kraus \& Hillenbrand 2008), the binary regime only encompasses separations of $\lesssim 2^{\prime}$. The observed scatter is unlikely to result from any distance dispersion of Taurus members $(\lesssim 15$ pc for members in similar parts of the cloud; Section 3.1) since it would yield a scatter of $\lesssim 0.2 \mathrm{mag}$ in $M_{\text {bol }}$ or $\sim 0.15$ dex in $\log \tau(0.20-0.25$ dex in $|\Delta \log \tau|$ for a pair of stars).

\subsection{Implications for (Multiple) Star Formation}

Our results for binary pair age differences are consistent with theoretical predictions for the timescale of local star formation (i.e., within one core) and global star formation (spanning the entire molecular cloud). If most binary systems in Taurus are coeval to within $<0.16$ dex (including observational uncertainties), then given its median age (1.8 $\pm 0.2 \mathrm{Myr}$; Appendix A), the formation times for binary components must differ by $\lesssim 0.7$ Myr. The expected timescale for an individual protostar to collapse after achieving supercriticality is the dynamical timescale ( 0.1-0.2 Myr; Shu et al. 1987); binary fragmentation is likely to occur during this collapse, so our limit is consistent with the predicted formation timescale. Recent simulations that exploit smoothed-particle hydrodynamic and $N$-body codes also suggest that most binary systems form within $\lesssim 0.5$ Myr (Delgado-Donate et al. 2004).

In contrast, the timescale for global star formation is likely to be much longer, representing either the turbulent dissipation timescale ( $\sim 1$ Myr; Ballesteros-Paredes et al. 1999; Elmegreen 2000) or the ambipolar diffusion timescale ( 310 Myr; Mouschovias 1976; Shu 1977). Our limit on the age dispersion of binary pairs is shorter than either timescale, while the overall age dispersion for unrelated pairs of stars is consistent with the ambipolar diffusion timescale, but only marginally with the turbulent dissipation timescale. We found a dispersion of $\sim 0.6$ dex in $|\Delta \log \tau|$ for random pairs, corresponding to a dispersion in $\log \tau$ of $\sim 0.4$ dex. For the median Taurus age of $\sim 1.8 \mathrm{Myr}$, this corresponds to a typical age range of 1-5 Myr.

If our results do reveal two distributions (one population that appears coeval and one that does not), then the number of systems in each distribution will allow a constraint on the fraction of binary systems that are genuinely coeval. Of the 36 pairs of stars that we considered, 24 are coeval to within $|\Delta \log \tau|<0.4$ dex, while the other 12 have ages that are more discrepant. This suggests that $\gtrsim 67_{-9}^{+7} \%$ of all binary systems are coeval with a dispersion of $\lesssim 0.16$ dex. However, many of the noncoeval pairs could be affected by systematic errors while being genuinely coeval, so this fraction is a lower limit. A more intensive study of the apparently noncoeval pairs should be a priority; as discussed above, some stars (such as V710 Tau C, DK Tau A, and XZ Tau B) already seem potentially affected by systematic errors and might be rejected from our sample based on additional follow-up observations.

Finally, our results indicate that the properties of a binary system correlate only modestly with the formation timescale; even extreme systems (with very wide separations or disparate masses) appear similarly coeval on average. Conversely, unbound pairs of stars that are only modestly separated $(\sim 1 \mathrm{pc})$ show the full age dispersion of the association. These trends strongly indicate that binary coevality is a natural result of the binary formation process itself, not a reflection of any trend for star formation to occur simultaneously within larger regions of the natal molecular cloud.

\section{SUMMARY}

We have studied the binary population of the Taurus-Auriga association in order to quantify the frequency and degree of noncoevality in young binary systems. After identifying and rejecting the systems that are known to be affected by systematic errors (such as further multiplicity or obscuration by circumstellar material), we used pre-main sequence evolutionary tracks to infer individual ages for the individual stars in binary systems, and hence the relative binary ages. We have found that the overall dispersion in the relative ages $(|\Delta \log \tau|)$ is 0.40 dex, though the distribution actually appears bimodal. Random pairs of Taurus members are coeval only to within $0.58 \mathrm{dex}$, suggesting that Taurus binaries are more coeval than the association as a whole.

The bimodality indicates that our sample is comprised of two populations, one with $\sim 2 / 3$ appearing to be coeval binaries 
with a dispersion of $\sigma_{|\Delta \log \tau|} \sim 0.16$ dex and the other with $\sim 1 / 3$ appearing to be systematically offset from coevality by $\sim 0.6$ dex. The noncoeval population shows no trends with respect to the system mass, separation, or mass ratio, which defies the predictions of many formation scenarios for noncoeval systems. We therefore suggest that the noncoeval population is comprised mainly of unrecognized hierarchical multiples, stars seen in scattered light, or stars with NIR disk excesses; identifying any truly noncoeval systems will require additional follow-up to rule out or correct for these explanations. The full range of apparent ages in our sample is $\sim 1$ dex, which suggests that a binary system tends to form in a very short period of time relative to the global star formation timescale for Taurus.

Finally, we found that the relative coevality of binary systems does not depend significantly on the system mass, mass ratio, or separation. However, any pair of Taurus members wider than $\sim 10^{\prime} \quad(\sim 0.7 \mathrm{pc})$ shows the full age spread of the association. This suggests that the enhanced coevality is seen only for binary systems and not for neighboring stars that formed from separate protostellar cores. The apparent coevality of a large fraction of our sample is also a partial endorsement of pre-main sequence isochrones. We did invoke several corrections to the model tracks, but any additional mass-dependent error in ages would cause systems with unequal mass ratios to appear less coeval, and we see no such trend to within the uncertainties in our results.

The authors thank R. White and G. Herczeg for helpful feedback on several of the ideas presented here, as well as E. Mamajek for a helpful discussion of Taurus membership issues. We also thank the referee for a prompt and helpful review. A. L. K. was supported by a NASA Origins grant to L. A. H. and by a SIM Science Study. This work makes use of data products from 2MASS, which is a joint project of the University of Massachusetts and the IPAC/Caltech, funded by NASA and the NSF. This work also made extensive use of the SIMBAD database, operated at CDS, Strasbourg, France.

\section{APPENDIX A}

\section{THE SINGLE STARS IN TAURUS}

The single stars of Taurus provide a useful check on the validity of our results, as well as providing their own constraints on its star formation history. In this appendix, we compile a sample of all stars that have a significant probability of being single (based on nondetections with one or more high-resolution imaging techniques). We then place these single stars on an HR diagram and estimate their ages and masses. Finally, we investigate the dependence of apparent age on stellar mass and location within the association.

\section{A.1. Sample}

We list our sample of apparently single stars in Table A1, including all of the references for our adopted parameters (singleness, spectral type, and extinction). We based our sample on the compilation of all Taurus members that we originally described in Kraus \& Hillenbrand 2007a), and then we omitted all stars that did not have at least one observation at high angular resolution. We then searched the literature for spectral types, requiring uncertainties of $\leqslant 1$ subclass for spectral types $>\mathrm{K} 0$ and $\leqslant 2$ subclasses for earlier-type stars.

Most stars have only been surveyed for multiplicity to a separation limit of 50-100 mas (7-15 AU), so close binary systems still contaminate this sample. However, we do not expect any mass-dependent systematic biases. The binary fraction drops significantly with declining primary mass (e.g., Kraus et al. $2006,2008)$, but much of that drop is seen among the wider binary systems (e.g., Kraus \& Hillenbrand 2009) that would have fallen outside our required sensitivity limit of $\lesssim 15-20 \mathrm{AU}$.

We inferred the fundamental properties of these stars (luminosity and temperature, then age and mass) using the methods described in Section 3. We list these properties in Table A1.

\section{A.2. The Ages of Single Taurus Members}

In Figure A1, we show the HR diagram for our sample of single Taurus members. The composition of our singlestar sample is significantly different from our binary sample, featuring many high-mass $\left(>1 M_{\odot}\right)$ and low-mass $\left(<0.3 M_{\odot}\right)$ members, but few members with intermediate masses. This difference in composition is driven largely by selection biases since few binaries at either extremum of mass have spatially resolved spectra. High-mass binaries in Taurus tend to be hierarchical multiples with additional components, while lowmass binaries were difficult to observe with spectroscopy before the recent advent of laser guide star AO. As a result, we must be very cautious in comparing the bulk properties of both samples.

We also note that many members fall below our designated lower edge of the Taurus sequence (the $5 \mathrm{Myr}$ isochrone) and might have erroneously low luminosities. Most of these members have not been well-studied (e.g., ITG33a and I04301+2608), so we cannot reject them with certainty, but their presence as extreme outliers in our plots invites skepticism. Most of the highest-mass members $\left(\mathrm{SpT} \leqslant \mathrm{K} 3 ; M \gtrsim 1 M_{\odot}\right)$ also fall systematically below the 1-2 Myr isochrone. A few of our binary sample members fall in this mass range, but those that do are either obviously erroneous (the HBC stars) or appear genuinely young. We have no satisfactory explanation for this discrepancy between the single stars and binary components since an error in our methods or in the underlying models should affect both populations equally, but the small number of high-mass stars in our binary sample suggests that the single-star sample might provide a more reliable indication of the true empirical isochrone.

In Figure A2, we show the model-derived age as a function of model-derived mass for our sample of single stars. Most stars seem to track the median age of Taurus, but as noted above, the highest-mass stars $\left(\gtrsim 1 M_{\odot}\right)$ appear systematically older. The brown dwarfs of Taurus $\left(M \lesssim 0.07 M_{\odot}\right)$ have very uncertain ages, so it is difficult to determine when they formed in relation to the stars. This uncertainty is driven by the physics of brown dwarf contraction, as isochrones at ages of $\lesssim 10$ Myr follow similar tracks in the HR diagram. If we only consider members with masses of $\sim 0.07-0.9 M_{\odot}$, then the median age of Taurus is $\log (\tau)=6.25 \pm 0.05 \mathrm{yr}(1.8 \pm 0.2 \mathrm{Myr})$.

Finally, in Figure A3, we show the spatial distribution of our single-star sample on the sky, with the position of each star color-coded according to its age, as well as the mean age for the eastern subgroup, southern subgroup, and the eastern and western halves of the central filaments. The eastern subgroup appears $\sim 4 \sigma$ older than the other subgroups (3.2 Myr, versus 1.4-1.9 Myr); if this age difference is genuine, then it suggests that star formation occurred first in Auriga, then in the rest of the association. However, this apparent age difference could also be a three-dimensional projection effect; the difference of $\sim 0.25$ dex in mean age could be explained if the distance to Auriga stars were $\sim 15 \%-20 \%$ larger than the mean distance 
Table A1

Single-star Sample: Observed Properties

\begin{tabular}{|c|c|c|c|c|c|c|c|c|c|c|}
\hline Name & $\begin{array}{l}\text { R.A. } \\
\quad(\mathrm{J} 2 \\
\end{array}$ & Decl. & $\begin{array}{c}J \\
(\mathrm{mag}) \\
\end{array}$ & SpT & $\begin{array}{c}A_{V} \\
(\mathrm{mag})\end{array}$ & $\begin{array}{l}T_{\text {eff }} \\
(\mathrm{K})\end{array}$ & $\begin{array}{c}M_{\text {bol }} \\
(\mathrm{mag})\end{array}$ & $\begin{array}{c}M \\
\left(M_{\odot}\right) \\
\end{array}$ & $\begin{array}{c}\log (\tau) \\
(\mathrm{yr})\end{array}$ & Refs \\
\hline 2MASSJ04080782+2807280 & 40807.82 & +280728.0 & 12.44 & $\mathrm{M} 3.75 \pm 0.25$ & 1.0 & $3305 \pm 35$ & 8.18 & $0.25_{-0.03}^{+0.03}$ & $7.04_{-0.17}^{+0.16}$ & 1,2 \\
\hline LkCa1 & 41314.14 & +281910.8 & 9.64 & $\mathrm{M} 4 \pm 0.5$ & 0.0 & $3270 \pm 70$ & 5.66 & $0.26_{-0.20}^{+0.13}$ & $5.70_{-0.32}^{+0.28}$ & 3,4 \\
\hline Anon1 & 41327.23 & +281624.8 & 8.83 & $\mathrm{M} 0 \pm 1$ & 3.6 & $3850 \pm 178$ & 3.59 & $0.65_{-0.03}^{+0.04}$ & $5.13_{-0.21}^{+0.20}$ & $3,5,6$ \\
\hline 2MASSJ04141188+2811535 & 41411.88 & +281153.5 & 13.16 & M6.25 \pm 0.25 & 0.7 & $2960 \pm 28$ & 9.08 & $0.069_{-0.006}^{+0.008}$ & $6.50_{-0.18}^{+0.19}$ & 1,7 \\
\hline FMTau & 41413.58 & +281249.2 & 10.33 & $\mathrm{M} 0 \pm 1$ & 1.9 & $3850 \pm 178$ & 5.56 & $0.68_{-0.08}^{+0.09}$ & $6.42_{-0.24}^{+0.23}$ & $3,4,6$ \\
\hline FNTau & 41414.59 & +282758.1 & 9.47 & $\mathrm{M} 5 \pm 0.5$ & 1.4 & $3125 \pm 70$ & 5.23 & $-0.20_{-1.10}^{+0.20}$ & $4.20_{-22.11}^{+0.97}$ & 3,8 \\
\hline CWTau & 41417.00 & +281057.8 & 9.56 & $\mathrm{~K} 3 \pm 1$ & 1.9 & $4730 \pm 155$ & 4.60 & $1.00_{-0.07}^{+0.22}$ & $6.39_{-0.21}^{+0.29}$ & $3,6,8$ \\
\hline CIDA-1 & 41417.61 & +280609.7 & 11.73 & M5.5 \pm 0.5 & 3.0 & $3060 \pm 68$ & 7.02 & $0.02_{-0.23}^{+0.15}$ & $4.40_{-3.98}^{+1.80}$ & $9,10,11$ \\
\hline MHO-1 & 41426.40 & +280559.7 & 11.52 & $\mathrm{M} 2.5 \pm 0.5$ & 5.7 & $3490 \pm 73$ & 5.90 & $0.49_{-0.07}^{+0.07}$ & $6.30_{-0.18}^{+0.20}$ & $6,10,12$ \\
\hline FPTau & 41447.31 & +264626.4 & 9.89 & $\mathrm{M} 4 \pm 0.5$ & 0.2 & $3270 \pm 70$ & 5.85 & $0.27_{-0.18}^{+0.11}$ & $5.82_{-0.31}^{+0.26}$ & 3,4 \\
\hline CXTau & 41447.86 & +264811.0 & 9.87 & $\mathrm{M} 2.5 \pm 0.5$ & 0.8 & $3490 \pm 73$ & 5.59 & $0.53_{-0.07}^{+0.05}$ & $6.15_{-0.18}^{+0.19}$ & 3,4 \\
\hline KPNO-Tau-1 & 41514.71 & +280009.6 & 15.10 & $\mathrm{M} 8.5 \pm 0.25$ & 0.4 & $2555 \pm 78$ & 11.13 & $0.023_{-0.006}^{+0.010}$ & $6.75_{-1.36}^{+0.40}$ & $13,14,15$ \\
\hline 2MASSJ04152409+2910434 & 41524.09 & +291043.4 & 13.68 & $\mathrm{M} 7 \pm 0.25$ & 2.0 & $2880 \pm 33$ & 9.27 & $0.053_{-0.005}^{+0.006}$ & $6.31_{-0.26}^{+0.26}$ & 1,2 \\
\hline 2MASSJ04161210+2756386 & 41612.10 & +275638.6 & 12.27 & $\mathrm{M} 4.75 \pm 0.25$ & 2.0 & $3160 \pm 38$ & 7.81 & $0.181_{-0.023}^{+0.022}$ & $6.55_{-0.14}^{+0.11}$ & 7,16 \\
\hline 2MASSJ04161885+2752155 & 41618.85 & +275215.5 & 12.55 & $\mathrm{M} 6.25 \pm 0.25$ & 1.0 & $2960 \pm 28$ & 8.39 & $0.080_{-0.008}^{+0.008}$ & $6.18_{-0.16}^{+0.16}$ & 1,2 \\
\hline 2MASSJ04163911+2858491 & 41639.11 & +285849.1 & 12.72 & $\mathrm{M} 5.5 \pm 0.25$ & 2.8 & $3060 \pm 38$ & 8.07 & $0.13_{-0.03}^{+0.02}$ & $6.44_{-0.18}^{+0.12}$ & 1,2 \\
\hline CYTau & 41733.73 & +282046.9 & 9.83 & $\mathrm{M} 1 \pm 0.5$ & 0.1 & $3705 \pm 73$ & 5.64 & $0.61_{-0.02}^{+0.04}$ & $6.35_{-0.20}^{+0.20}$ & 3,8 \\
\hline KPNO-Tau-10 & 41749.55 & +28 1331.9 & 11.89 & M5 \pm 0.25 & 0.0 & $3125 \pm 33$ & 8.02 & $0.15_{-0.02}^{+0.02}$ & $6.55_{-0.13}^{+0.11}$ & $1,14,17$ \\
\hline V410-Xray1 & 41749.65 & +282936.3 & 11.02 & $\mathrm{M} 3.75 \pm 0.25$ & 0.9 & $3305 \pm 35$ & 6.78 & $0.29_{-0.02}^{+0.03}$ & $6.36_{-0.14}^{+0.16}$ & $6,10,18$ \\
\hline V410-Anon13 & 41817.11 & +282841.9 & 12.96 & $\mathrm{M} 6 \pm 0.5$ & 3.8 & $2990 \pm 63$ & 8.02 & $0.09_{-0.02}^{+0.03}$ & $6.12_{-0.25}^{+0.31}$ & $6,12,15$ \\
\hline KPNO-Tau-11 & 41830.31 & +274320.8 & 11.89 & $\mathrm{M} 5.5 \pm 0.25$ & 0.0 & $3060 \pm 38$ & 8.01 & $0.13_{-0.03}^{+0.02}$ & $6.42_{-0.17}^{+0.12}$ & 1,17 \\
\hline KPNO-Tau-2 & 41851.16 & +281433.2 & 13.92 & $\mathrm{M} 7.5 \pm 0.25$ & 0.4 & $2795 \pm 45$ & 9.96 & $0.040_{-0.005}^{+0.007}$ & $6.71_{-0.67}^{+0.13}$ & $13,14,15$ \\
\hline НBC376 & 41851.70 & +172316.6 & 10.03 & $\mathrm{~K} 7 \pm 1$ & 0.0 & $4060 \pm 250$ & 5.66 & $0.79_{-0.13}^{+0.10}$ & $6.63_{-0.26}^{+0.27}$ & 3,4 \\
\hline I04158+2805 & 41858.14 & +28 1223.5 & 13.78 & $\mathrm{M} 6 \pm 1$ & 8.6 & $2990 \pm 123$ & 7.50 & $0.10_{-0.04}^{+0.07}$ & $5.91_{-0.88}^{+0.45}$ & 10,19 \\
\hline KPNO-Tau-12 & 41901.27 & +280248.7 & 16.31 & $\mathrm{M} 9 \pm 0.25$ & 0.5 & $2400 \pm 75$ & 12.31 & $0.032_{-0.010}^{+0.007}$ & $7.46_{-0.28}^{+0.23}$ & 15,17 \\
\hline V410-Xray5a & 41901.98 & +282233.2 & 11.99 & $\mathrm{M} 5.5 \pm 0.5$ & 2.6 & $3060 \pm 68$ & 7.41 & $0.14_{-0.12}^{+0.04}$ & $6.20_{-1.88}^{+0.17}$ & $6,10,12$ \\
\hline ВРТau & 41915.84 & +290626.9 & 9.10 & $\mathrm{~K} 7 \pm 1$ & 0.5 & $4060 \pm 250$ & 4.59 & $0.73_{-0.08}^{+0.09}$ & $5.94_{-0.29}^{+0.25}$ & 3,5 \\
\hline 2MASSJ04202555+2700355 & 42025.55 & +270035.5 & 12.86 & M5.25 \pm 0.25 & 2.0 & $3095 \pm 33$ & 8.42 & $0.123_{-0.014}^{+0.018}$ & $6.62_{-0.11}^{+0.11}$ & 1,7 \\
\hline$J 2-157$ & 42052.73 & +174641.5 & 11.62 & $\mathrm{M} 5.5 \pm 0.5$ & 0.0 & $3060 \pm 68$ & 7.74 & $0.14_{-0.04}^{+0.03}$ & $6.32_{-0.35}^{+0.17}$ & 3,10 \\
\hline CFHT-Tau-19 & 42107.95 & +270220.4 & 13.85 & $\mathrm{M} 5.25 \pm 0.25$ & 7.3 & $3095 \pm 33$ & 7.95 & $0.145_{-0.018}^{+0.020}$ & $6.46_{-0.13}^{+0.12}$ & 14,16 \\
\hline 2MASSJ04213460+2701388 & 42134.60 & +270138.8 & 11.90 & $\mathrm{M} 5.5 \pm 0.25$ & 1.8 & $3060 \pm 38$ & 7.53 & $0.14_{-0.05}^{+0.02}$ & $6.24_{-0.73}^{+0.13}$ & 7,16 \\
\hline CFHT-Tau-10 & 42146.31 & +265929.6 & 13.82 & $\mathrm{M} 5.75 \pm 0.25$ & 2.0 & $3020 \pm 35$ & 9.38 & $0.084_{-0.012}^{+0.012}$ & $6.88_{-0.22}^{+0.45}$ & 1,2 \\
\hline 2MASSJ04215450+2652315 & 42154.50 & +265231.5 & 15.54 & $\mathrm{M} 8.5 \pm 0.25$ & 1.0 & $2555 \pm 78$ & 11.40 & $0.027_{-0.009}^{+0.012}$ & $7.09_{-0.85}^{+0.23}$ & 1,2 \\
\hline DETau & 42155.64 & +275506.1 & 9.18 & $\mathrm{M} 2 \pm 0.5$ & 0.6 & $3560 \pm 70$ & 4.93 & $0.59_{-0.01}^{+0.04}$ & $5.82_{-0.20}^{+0.19}$ & 3,8 \\
\hline RYTau & 42157.40 & +282635.5 & 7.16 & $\mathrm{~K} 1 \pm 1$ & 1.8 & $5080 \pm 175$ & 2.09 & $2.1_{-0.6}^{+0.6}$ & $5.61_{-0.35}^{+0.28}$ & 3,8 \\
\hline HD283572 & 42158.84 & +281806.6 & 7.42 & $\mathrm{G} 5 \pm 2$ & 0.4 & $5770 \pm 98$ & 2.54 & $2.0_{-0.2}^{+0.4}$ & $6.62_{-0.25}^{+0.10}$ & 3,5 \\
\hline CFHT-Tau-14 & 42216.44 & +254911.8 & 13.06 & $\mathrm{M} 7.75 \pm 0.25$ & 0.5 & $2750 \pm 43$ & 9.06 & $0.036_{-0.006}^{+0.005}$ & $4.19_{-2.14}^{+1.12}$ & $1,2,14$ \\
\hline CFHT-Tau-21 & 42216.76 & +265457.1 & 11.58 & $\mathrm{M} 1.5 \pm 0.25$ & 3.0 & $3630 \pm 38$ & 6.63 & $0.58_{-0.03}^{+0.02}$ & $6.91_{-0.18}^{+0.18}$ & 2,16 \\
\hline 2MJ04230607+2801194 & 42306.07 & +280119.4 & 12.24 & $\mathrm{M} 6.25 \pm 0.25$ & 0.0 & $2960 \pm 28$ & 8.36 & $0.080_{-0.008}^{+0.008}$ & $6.17_{-0.16}^{+0.16}$ & 1,20 \\
\hline CFHT-Tau-9 & 42426.46 & +264950.4 & 12.88 & $\mathrm{M} 5.75 \pm 0.25$ & 0.5 & $3020 \pm 35$ & 8.86 & $0.087_{-0.010}^{+0.012}$ & $6.57_{-0.17}^{+0.21}$ & 1,2 \\
\hline IPTau & 42457.08 & +271156.5 & 9.78 & $\mathrm{M} 0 \pm 1$ & 0.2 & $3850 \pm 178$ & 5.47 & $0.68_{-0.08}^{+0.08}$ & $6.37_{-0.24}^{+0.23}$ & 3,5 \\
\hline KPNO-Tau-3 & 42629.39 & +262413.8 & 13.32 & $\mathrm{M} 6 \pm 0.25$ & 1.6 & $2990 \pm 30$ & 8.98 & $0.077_{-0.008}^{+0.009}$ & $6.55_{-0.17}^{+0.17}$ & 13,15 \\
\hline KPNO-Tau-13 & 42657.33 & +260628.4 & 11.28 & M5 \pm 0.25 & 2.5 & $3125 \pm 33$ & 6.71 & $0.12_{-0.07}^{+0.08}$ & $5.84_{-0.51}^{+0.31}$ & 10,17 \\
\hline НBC388 & 42710.56 & +175042.6 & 8.79 & $\mathrm{~K} 1 \pm 1$ & 0.1 & $5080 \pm 175$ & 4.20 & $1.42_{-0.13}^{+0.12}$ & $6.63_{-0.26}^{+0.24}$ & 3,5 \\
\hline KPNO-Tau-4 & 42728.00 & +261205.3 & 15.00 & $\mathrm{M} 9.5 \pm 0.25$ & 2.5 & $2245 \pm 80$ & 10.46 & $-0.015_{-0.006}^{+0.011}$ & $3.45_{-0.58}^{+1.41}$ & $13,14,15$ \\
\hline 2MASSJ04290068+2755033 & 42900.68 & +275503.3 & 14.02 & $\mathrm{M} 8.25 \pm 0.25$ & 0.0 & $2630 \pm 578$ & 10.16 & $0.02_{-0.17}^{+0.13}$ & $4.46_{-16.38}^{+3.38}$ & 1,2 \\
\hline KPNO-Tau-5 & 42945.68 & +263046.8 & 12.64 & $\mathrm{M} 7.5 \pm 0.25$ & 0.0 & $2795 \pm 45$ & 8.78 & $0.043_{-0.006}^{+0.008}$ & $4.77_{-1.44}^{+1.00}$ & 13,15 \\
\hline IQTau & 42951.56 & +260644.9 & 9.42 & $\mathrm{M} 0.5 \pm 0.5$ & 1.3 & $3775 \pm 73$ & 4.87 & $0.64_{-0.02}^{+0.03}$ & $5.93_{-0.18}^{+0.19}$ & 3,5 \\
\hline CFHT-Tau-20 & 42959.51 & +243307.9 & 11.68 & M5 \pm 0.25 & 2.2 & $3125 \pm 33$ & 7.19 & $0.18_{-0.04}^{+0.02}$ & $6.21_{-0.23}^{+0.15}$ & 2,16 \\
\hline KPNO-Tau-6 & 43007.24 & +260820.8 & 15.00 & $\mathrm{M} 8.5 \pm 0.25$ & 0.9 & $2555 \pm 78$ & 10.90 & $0.021_{-0.007}^{+0.007}$ & $5.98_{-1.92}^{+0.99}$ & $13,14,15$ \\
\hline CFHT-Tau-16 & 43023.65 & +235913.0 & 14.96 & $\mathrm{M} 8.25 \pm 0.25$ & 0.0 & $2630 \pm 578$ & 11.10 & $0.03_{-0.11}^{+0.12}$ & $7.00_{-11.40}^{+1.37}$ & 1,2 \\
\hline KPNO-Tau-7 & 43057.19 & +25 56 39.5 & 14.52 & $\mathrm{M} 8.25 \pm 0.25$ & 0.0 & $2630 \pm 578$ & 10.66 & $0.03_{-0.14}^{+0.12}$ & $6.55_{-14.43}^{+1.53}$ & 13,15 \\
\hline JH56 & 43114.44 & +271018.0 & 9.70 & $\mathrm{M} 0.5 \pm 0.5$ & 1.1 & $3775 \pm 73$ & 5.21 & $0.64_{-0.03}^{+0.03}$ & $6.13_{-0.19}^{+0.20}$ & 3,10 \\
\hline MHO-9 & 43115.78 & +182007.2 & 11.21 & $\mathrm{M} 5 \pm 0.5$ & 2.2 & $3125 \pm 70$ & 6.73 & $0.10_{-0.40}^{+0.10}$ & $5.86_{-6.59}^{+0.34}$ & $6,9,10$ \\
\hline 2MASSJ04311907+2335047 & 43119.07 & +233504.7 & 13.51 & $\mathrm{M} 7.75 \pm 0.25$ & 0.5 & $2750 \pm 43$ & 9.51 & $0.036_{-0.005}^{+0.005}$ & $5.28_{-1.61}^{+0.90}$ & 1,2 \\
\hline MHO-4 & 43124.06 & +180021.5 & 11.66 & $\mathrm{M} 7 \pm 0.5$ & 1.0 & $2880 \pm 70$ & 7.53 & $0.07_{-0.02}^{+0.03}$ & $5.19_{-1.44}^{+0.65}$ & $6,9,15$ \\
\hline CFHT-Tau-13 & 43126.69 & +270318.8 & 14.83 & $\mathrm{M} 7.5 \pm 0.25$ & 0.5 & $2795 \pm 45$ & 10.84 & $0.049_{-0.010}^{+0.011}$ & $7.14_{-0.21}^{+0.27}$ & 1,2 \\
\hline LkHa358 & 43136.13 & +181343.3 & 12.80 & $\mathrm{M} 5.5 \pm 0.5$ & 13.5 & $3060 \pm 68$ & 5.18 & $-0.9_{-0.5}^{+1.0}$ & $-9.28_{-8.92}^{+14.39}$ & $3,6,10$ \\
\hline
\end{tabular}


Table A1

(Continued)

\begin{tabular}{|c|c|c|c|c|c|c|c|c|c|c|}
\hline Name & \multicolumn{2}{|c|}{$(\mathrm{J} 2000)$} & $\begin{array}{c}J \\
\text { (mag) } \\
\end{array}$ & SpT & $\begin{array}{c}A_{V} \\
(\mathrm{mag}) \\
\end{array}$ & $\begin{array}{l}T_{\text {eff }} \\
(\mathrm{K}) \\
\end{array}$ & $\begin{array}{c}M_{\text {bol }} \\
(\mathrm{mag}) \\
\end{array}$ & $\begin{array}{c}M \\
\left(M_{\odot}\right) \\
\end{array}$ & $\begin{array}{c}\log (\tau) \\
(\mathrm{yr})\end{array}$ & Refs \\
\hline HLTau & 43138.44 & +181357.7 & 10.62 & $\mathrm{~K} 5 \pm 1$ & 7.4 & $4350 \pm 265$ & 4.25 & $0.83_{-0.10}^{+0.11}$ & $5.93_{-0.25}^{+0.24}$ & 8,19 \\
\hline $\mathrm{J} 1-665$ & 43158.44 & +254329.9 & 10.59 & $\mathrm{M} 5 \pm 0.5$ & 1.0 & $3125 \pm 70$ & 6.45 & $0.10_{-0.60}^{+0.10}$ & $5.55_{-9.46}^{+0.50}$ & 3,10 \\
\hline 2MASSJ04320329+2528078 & 43203.29 & +252807.8 & 11.72 & $\mathrm{M} 6.25 \pm 0.25$ & 0.0 & $2960 \pm 28$ & 7.84 & $0.089_{-0.008}^{+0.008}$ & $5.95_{-0.15}^{+0.16}$ & 1,2 \\
\hline L1551-51 & 43209.27 & +175722.8 & 9.70 & $\mathrm{~K} 7 \pm 1$ & 0.0 & $4060 \pm 250$ & 5.33 & $0.76_{-0.11}^{+0.11}$ & $6.42_{-0.26}^{+0.25}$ & $3,4,6$ \\
\hline Haro6-13 & 43215.41 & +242859.7 & 11.24 & $\mathrm{M} 0 \pm 0.5$ & 11.9 & $3850 \pm 90$ & 3.72 & $0.653_{-0.017}^{+0.018}$ & $5.22_{-0.20}^{+0.20}$ & 8,19 \\
\hline MHO-5 & 43216.07 & +181246.4 & 11.07 & $\mathrm{M} 7 \pm 0.5$ & 0.1 & $2880 \pm 70$ & 7.18 & $0.07_{-0.02}^{+0.03}$ & $4.97_{-1.63}^{+0.75}$ & $6,9,15$ \\
\hline CFHT-Tau-7 & 43217.86 & +242215.0 & 11.54 & $\mathrm{M} 5.75 \pm 0.25$ & 0.0 & $3020 \pm 35$ & 7.65 & $0.12_{-0.03}^{+0.02}$ & $6.21_{-0.98}^{+0.13}$ & 2,10 \\
\hline MHO-6 & 43222.11 & +182742.6 & 11.71 & $\mathrm{M} 4.75 \pm 0.25$ & 0.9 & $3160 \pm 38$ & 7.57 & $0.19_{-0.02}^{+0.02}$ & $6.46_{-0.16}^{+0.14}$ & $6,10,13$ \\
\hline 2MASSJ04322329+2403013 & 43223.29 & +240301.3 & 12.34 & $\mathrm{M} 7.75 \pm 0.25$ & 0.0 & $2750 \pm 43$ & 8.48 & $0.037_{-0.006}^{+0.006}$ & $2.76_{-2.87}^{+1.44}$ & 1,2 \\
\hline MHO-7 & 43226.28 & +182752.1 & 11.11 & $\mathrm{M} 5.25 \pm 0.25$ & 0.4 & $3095 \pm 33$ & 7.12 & $0.15_{-0.08}^{+0.03}$ & $6.12_{-0.99}^{+0.16}$ & $6,10,13$ \\
\hline FYTau & 43230.58 & +241957.3 & 9.98 & $\mathrm{~K} 7 \pm 1$ & 3.5 & $4060 \pm 250$ & 4.65 & $0.74_{-0.09}^{+0.09}$ & $5.98_{-0.29}^{+0.25}$ & 3,4 \\
\hline FZTau & 43231.76 & +242003.0 & 9.90 & $\mathrm{M} 0 \pm 1$ & 3.6 & $3850 \pm 178$ & 4.67 & $0.66_{-0.05}^{+0.06}$ & $5.84_{-0.20}^{+0.24}$ & $8,13,21$ \\
\hline L1551-55 & 43243.73 & +180256.3 & 10.16 & $\mathrm{~K} 7 \pm 1$ & 0.7 & $4060 \pm 250$ & 5.60 & $0.78_{-0.12}^{+0.11}$ & $6.59_{-0.26}^{+0.27}$ & 3,22 \\
\hline KPNO-Tau-14 & 43307.81 & +261606.6 & 11.91 & $\mathrm{M} 6 \pm 0.25$ & 3.1 & $2990 \pm 30$ & 7.17 & $0.11_{-0.07}^{+0.01}$ & $5.77_{-2.31}^{+0.16}$ & 15,17 \\
\hline V830Tau & 43310.03 & +243343.4 & 9.32 & $\mathrm{~K} 7 \pm 1$ & 0.3 & $4060 \pm 250$ & 4.87 & $0.74_{-0.09}^{+0.10}$ & $6.12_{-0.27}^{+0.26}$ & 3,5 \\
\hline $\mathrm{I} 04303+2240$ & 43319.07 & +224634.2 & 11.103 & $\mathrm{M} 0.5 \pm 1$ & 11.7 & $3775 \pm 163$ & 3.67 & $0.64_{-0.03}^{+0.03}$ & $5.20_{-0.19}^{+0.18}$ & 10,19 \\
\hline GITau & 43334.06 & +2421 17.0 & 9.34 & $\mathrm{~K} 6 \pm 1$ & 0.9 & $4350 \pm 265$ & 4.78 & $0.85_{-0.10}^{+0.11}$ & $6.25_{-0.25}^{+0.27}$ & 3,8 \\
\hline DLTau & 43339.06 & +252038.2 & 9.63 & $\mathrm{~K} 7 \pm 1$ & 1.7 & $4060 \pm 250$ & 4.79 & $0.74_{-0.09}^{+0.09}$ & $6.07_{-0.28}^{+0.25}$ & $3,4,23$ \\
\hline 2MASSJ04334291+2526470 & 43342.91 & +252647.0 & 14.64 & $\mathrm{M} 8.75 \pm 0.25$ & 0.0 & $2475 \pm 78$ & 10.78 & $0.014_{-0.007}^{+0.006}$ & $5.74_{-1.48}^{+0.33}$ & 1,2 \\
\hline DMTau & 43348.72 & +181010.0 & 10.44 & $\mathrm{M} 1 \pm 0.5$ & 0.0 & $3705 \pm 73$ & 6.28 & $0.63_{-0.04}^{+0.05}$ & $6.76_{-0.20}^{+0.20}$ & 3,4 \\
\hline CITau & 43352.00 & +225030.2 & 9.48 & $\mathrm{~K} 7 \pm 1$ & 1.8 & $4060 \pm 250$ & 4.62 & $0.74_{-0.08}^{+0.09}$ & $5.96_{-0.29}^{+0.25}$ & 3,8 \\
\hline JH108 & 43410.99 & +225144.5 & 10.60 & $\mathrm{M} 1 \pm 0.5$ & 1.5 & $3705 \pm 73$ & 6.03 & $0.62_{-0.04}^{+0.05}$ & $6.60_{-0.20}^{+0.20}$ & 3,24 \\
\hline CFHT-Tau-1 & 43415.27 & +225031.0 & 13.74 & $\mathrm{M} 7 \pm 0.25$ & 3.1 & $2880 \pm 33$ & 9.02 & $0.055_{-0.005}^{+0.006}$ & $6.14_{-0.27}^{+0.25}$ & 15,25 \\
\hline AATau & 43455.42 & +242853.2 & 9.44 & $\mathrm{~K} 7 \pm 1$ & 0.5 & $4060 \pm 250$ & 4.93 & $0.74_{-0.09}^{+0.10}$ & $6.16_{-0.27}^{+0.26}$ & 3,4 \\
\hline HOTau & 43520.20 & +223214.6 & 11.20 & M0.5 \pm 0.5 & 1.1 & $3775 \pm 73$ & 6.69 & $0.69_{-0.06}^{+0.04}$ & $7.10_{-0.22}^{+0.23}$ & 3,24 \\
\hline DNTau & 43527.37 & +241458.9 & 9.14 & $\mathrm{M} 0 \pm 0.5$ & 1.9 & $3850 \pm 90$ & 4.37 & $0.66_{-0.03}^{+0.03}$ & $5.65_{-0.20}^{+0.20}$ & 5,19 \\
\hline KPNO-Tau-8 & 43541.84 & +223411.6 & 12.95 & $\mathrm{M} 5.75 \pm 0.25$ & 0.5 & $3020 \pm 35$ & 8.92 & $0.086_{-0.010}^{+0.011}$ & $6.60_{-0.17}^{+0.24}$ & 13,15 \\
\hline KPNO-Tau-9 & 43551.43 & +224911.9 & 15.48 & $\mathrm{M} 8.5 \pm 0.25$ & 0.0 & $2555 \pm 78$ & 11.62 & $0.033_{-0.013}^{+0.011}$ & $7.24_{-0.43}^{+0.25}$ & 13,15 \\
\hline HPTau-G2 & 43554.15 & +225413.5 & 8.10 & $\mathrm{G} 0 \pm 2$ & 2.1 & $6030 \pm 170$ & 2.67 & $1.83_{-0.07}^{+0.07}$ & $6.75_{-0.05}^{+0.04}$ & 3,4 \\
\hline CFHT-Tau-2 & 43610.39 & +225956.0 & 13.76 & $\mathrm{M} 7.5 \pm 0.25$ & 2.0 & $2795 \pm 45$ & 9.34 & $0.041_{-0.005}^{+0.007}$ & $5.68_{-1.03}^{+0.63}$ & 13,15 \\
\hline LkCa14 & 43619.09 & +254259.0 & 9.34 & $\mathrm{M} 0 \pm 1$ & 0.0 & $3850 \pm 178$ & 5.10 & $0.67_{-0.06}^{+0.07}$ & $6.12_{-0.22}^{+0.24}$ & 3,4 \\
\hline CFHT-Tau-3 & 43638.94 & +225811.9 & 13.73 & $\mathrm{M} 7.75 \pm 0.25$ & 1.0 & $2750 \pm 43$ & 9.59 & $0.036_{-0.005}^{+0.004}$ & $5.46_{-1.53}^{+0.86}$ & 13,15 \\
\hline 2MASSJ04380084+2558572 & 43800.84 & +255857.2 & 11.54 & $\mathrm{M} 7.25 \pm 0.25$ & 0.6 & $2840 \pm 43$ & 7.51 & $0.059_{-0.010}^{+0.010}$ & $4.72_{-1.82}^{+0.52}$ & 7,16 \\
\hline GMTau & 43821.34 & +260913.7 & 12.80 & $\mathrm{M} 6.5 \pm 0.5$ & 2.0 & $2935 \pm 55$ & 8.38 & $0.074_{-0.013}^{+0.015}$ & $6.07_{-0.38}^{+0.25}$ & $9,11,15$ \\
\hline DOTau & 43828.58 & +261049.4 & 9.47 & $\mathrm{M} 0 \pm 1$ & 2.6 & $3850 \pm 178$ & 4.50 & $0.66_{-0.05}^{+0.06}$ & $5.73_{-0.20}^{+0.23}$ & 3,8 \\
\hline SCHJ0439016+2336030 & 43901.60 & +233603.0 & 11.34 & $\mathrm{M} 6 \pm 0.25$ & 0.0 & $2990 \pm 30$ & 7.45 & $0.10_{-0.03}^{+0.01}$ & $5.89_{-1.02}^{+0.25}$ & $1,11,26$ \\
\hline CIDA-13 & 43915.86 & +303207.4 & 12.68 & $\mathrm{M} 3.5 \pm 0.5$ & 0.4 & $3340 \pm 73$ & 8.59 & $0.25_{-0.05}^{+0.05}$ & $7.31_{-0.23}^{+0.22}$ & 10,27 \\
\hline LkCa15 & 43917.80 & +222103.5 & 9.42 & $\mathrm{~K} 5 \pm 1$ & 0.6 & $4350 \pm 265$ & 4.93 & $0.85_{-0.10}^{+0.11}$ & $6.35_{-0.26}^{+0.26}$ & 3,4 \\
\hline CFHT-Tau-4 & 43947.48 & +260140.8 & 12.17 & $\mathrm{M} 7 \pm 0.25$ & 3.0 & $2880 \pm 33$ & 7.48 & $0.068_{-0.007}^{+0.012}$ & $5.16_{-0.36}^{+0.39}$ & $13,15,25$ \\
\hline I04370+2559 & 44008.00 & +260525.4 & 12.41 & $\mathrm{M} 4.75 \pm 0.25$ & 10.0 & $3160 \pm 38$ & 5.75 & $-0.05_{-0.09}^{+0.13}$ & $5.24_{-0.60}^{+0.35}$ & 2,10 \\
\hline $\mathrm{I} 04385+2550$ & 44138.82 & +255626.8 & 11.85 & $\mathrm{M} 0.5 \pm 0.5$ & 10.2 & $3775 \pm 73$ & 4.83 & $0.64_{-0.02}^{+0.03}$ & $5.91_{-0.18}^{+0.19}$ & 10,19 \\
\hline CIDA-7 & 44221.02 & +252034.4 & 11.40 & $\mathrm{M} 4.75 \pm 0.25$ & 1.0 & $3160 \pm 38$ & 7.22 & $0.199_{-0.023}^{+0.021}$ & $6.29_{-0.15}^{+0.16}$ & 2,10 \\
\hline DPTau & 44237.70 & +251537.5 & 11.00 & $\mathrm{M} 0 \pm 1$ & 6.3 & $3850 \pm 178$ & 5.02 & $0.67_{-0.06}^{+0.07}$ & $6.07_{-0.22}^{+0.24}$ & 4,19 \\
\hline GOTau & 44303.09 & +252018.8 & 10.71 & $\mathrm{M} 0 \pm 1$ & 1.2 & $3850 \pm 178$ & 6.15 & $0.72_{-0.11}^{+0.09}$ & $6.81_{-0.25}^{+0.26}$ & 3,4 \\
\hline 2MASSJ04442713+2512164 & 44427.13 & +251216.4 & 12.19 & M7.25 \pm 0.25 & 0.0 & $2840 \pm 43$ & 8.33 & $0.054_{-0.008}^{+0.008}$ & $5.37_{-1.22}^{+0.41}$ & 7,16 \\
\hline DQTau & 44653.05 & +170000.2 & 9.51 & $\mathrm{M} 0 \pm 1$ & 1.0 & $3850 \pm 178$ & 5.00 & $0.67_{-0.06}^{+0.07}$ & $6.06_{-0.22}^{+0.24}$ & 3,4 \\
\hline DRTau & 44706.21 & +165842.8 & 8.84 & $\mathrm{~K} 7 \pm 1$ & 3.2 & $4060 \pm 250$ & 3.59 & $0.70_{-0.06}^{+0.09}$ & $5.29_{-0.36}^{+0.32}$ & $3,5,23$ \\
\hline DSTau & 44748.59 & +292511.2 & 9.47 & $\mathrm{~K} 5 \pm 1$ & 0.3 & $4350 \pm 265$ & 5.06 & $0.86_{-0.11}^{+0.11}$ & $6.44_{-0.26}^{+0.25}$ & 3,4 \\
\hline GMAur & 45510.98 & +302159.5 & 9.34 & $\mathrm{~K} 3 \pm 1$ & 0.1 & $4730 \pm 155$ & 4.88 & $1.02_{-0.08}^{+0.16}$ & $6.58_{-0.23}^{+0.26}$ & 3,4 \\
\hline 2MASSJ04552333+3027366 & 45523.33 & +302736.6 & 13.07 & M6.25 \pm 0.25 & 0.0 & $2960 \pm 28$ & 9.19 & $0.068_{-0.006}^{+0.007}$ & $6.56_{-0.19}^{+0.19}$ & 1,7 \\
\hline LkCa19 & 45536.96 & +301755.3 & 8.87 & $\mathrm{~K} 0 \pm 2$ & 0.0 & $5250 \pm 335$ & 4.27 & $1.35_{-0.16}^{+0.19}$ & $6.84_{-0.38}^{+0.27}$ & 3,4 \\
\hline 2MASSJ04554046+3039057 & 45540.46 & +303905.7 & 12.71 & M5.25 \pm 0.25 & 0.3 & $3095 \pm 33$ & 8.76 & $0.110_{-0.011}^{+0.0 .10}$ & $6.72_{-0.10}^{+0.30}$ & 1,7 \\
\hline 2MASSJ04554535+3019389 & 45545.35 & +301938.9 & 11.44 & $\mathrm{M} 4.75 \pm 0.25$ & 0.0 & $3160 \pm 38$ & 7.54 & $0.192_{-0.021}^{+0.020}$ & $6.44_{-0.16}^{+0.14}$ & 7,16 \\
\hline 2MASSJ04554970+3019400 & 45549.70 & +301940.0 & 12.81 & $\mathrm{M} 6 \pm 0.25$ & 0.0 & $2990 \pm 30$ & 8.92 & $0.078_{-0.008}^{+0.009}$ & $6.52_{-0.17}^{+0.17}$ & 1,7 \\
\hline 2MASSJ04555289+3006523 & 45552.89 & +300652.3 & 11.64 & $\mathrm{M} 5.25 \pm 0.25$ & 0.0 & $3095 \pm 33$ & 7.76 & $0.152_{-0.018}^{+0.019}$ & $6.40_{-0.13}^{+0.11}$ & 1,7 \\
\hline 2MASSJ04555637+3049375 & 45556.37 & +304937.5 & 12.00 & $\mathrm{M} 5 \pm 0.25$ & 0.4 & $3125 \pm 33$ & 8.03 & $0.15_{-0.02}^{+0.02}$ & $6.56_{-0.13}^{+0.11}$ & 1,7 \\
\hline SUAur & 45559.38 & +303401.6 & 7.20 & $\mathrm{G} 2 \pm 2$ & 0.9 & $5860 \pm 115$ & 2.11 & $2.3_{-0.3}^{+0.0 .3}$ & $6.39_{-0.21}^{+0.19}$ & 3,8 \\
\hline 2MASSJ04574903+3015195 & 45749.03 & +301519.5 & 15.77 & M9.25 \pm 0.25 & 0.0 & $2325 \pm 78$ & 11.91 & $0.016_{-0.005}^{+0.011}$ & $6.54_{-0.55}^{+0.76}$ & 1,7 \\
\hline
\end{tabular}


Table A1

(Continued)

\begin{tabular}{|c|c|c|c|c|c|c|c|c|c|c|}
\hline Name & R.A. & De) Decl. & $\begin{array}{c}J \\
(\mathrm{mag}) \\
\end{array}$ & $\mathrm{SpT}$ & $\begin{array}{c}A_{V} \\
(\mathrm{mag}) \\
\end{array}$ & $\begin{array}{l}T_{\text {eff }} \\
(\mathrm{K})\end{array}$ & $\begin{array}{c}M_{\text {bol }} \\
(\mathrm{mag})\end{array}$ & $\begin{array}{c}M \\
\left(M_{\odot}\right) \\
\end{array}$ & $\begin{array}{c}\log (\tau) \\
(\mathrm{yr})\end{array}$ & Refs \\
\hline V836Tau & 50306.60 & +252319.7 & 9.92 & $\mathrm{~K} 7 \pm 1$ & 1.7 & $4060 \pm 250$ & 5.08 & $0.75_{-0.10}^{+0.10}$ & $6.26_{-0.26}^{+0.26}$ & 4,19 \\
\hline CIDA-8 & 50441.40 & +250954.4 & 10.92 & $\mathrm{M} 3.5 \pm 0.5$ & 3.0 & $3340 \pm 73$ & 6.09 & $0.36_{-0.08}^{+0.06}$ & $6.13_{-0.23}^{+0.17}$ & 3,10 \\
\hline CIDA-10 & 50616.75 & +244610.2 & 10.80 & $\mathrm{M} 4 \pm 0.5$ & 0.5 & $3270 \pm 70$ & 6.68 & $0.27_{-0.04}^{+0.04}$ & $6.25_{-0.20}^{+0.19}$ & 3,10 \\
\hline $\mathrm{RX} 05072+2437$ & 50712.07 & +243716.4 & 10.14 & $\mathrm{~K} 6 \pm 0.5$ & 0.9 & $4350 \pm 133$ & 5.56 & $0.90_{-0.06}^{+0.04}$ & $6.77_{-0.22}^{+0.25}$ & $10,13,27$ \\
\hline CIDA-12 & 50754.97 & +250015.6 & 11.42 & $\mathrm{M} 4 \pm 0.5$ & 0.8 & $3270 \pm 70$ & 7.22 & $0.26_{-0.04}^{+0.04}$ & $6.51_{-0.20}^{+0.19}$ & 3,10 \\
\hline
\end{tabular}

Notes.

a Some systems that sit extremely high or low in the HR diagram exceed the limits of the models and have nonphysical derived quantities (i.e., negative masses).

References. (1) A. Kraus et al. 2009, in preparation; (2) Luhman 2006; (3) Kenyon \& Hartmann 1995; (4) Leinert et al. 1993; (5) Tanner et al. 2007; (6) Luhman et al. 2000; (7) Luhman 2004; (8) Ghez et al. 1993; (9) White \& Basri 2003; (10) R. White et al. 2009, in preparation; (11) Herczeg \& Hillenbrand 2008; (12) Briceño et al. 1998; (13) Briceño et al. 2002; (14) Guieu et al. 2006; (15) Kraus et al. 2006; (16) Konopacky et al. 2007; (17) Luhman et al. 2003; (18) Strom \& Strom 1994; (19) White \& Hillenbrand 2004; (20) Luhman et al. 2006; (21) Hartigan et al. 1994; (22) Sartoretti et al. 1998; (23) Hartigan et al. 1995; (24) Simon et al. 1995; (25) Martín et al. 2001; (26) Slesnick et al. 2006; (27) Briceño et al. 1999.

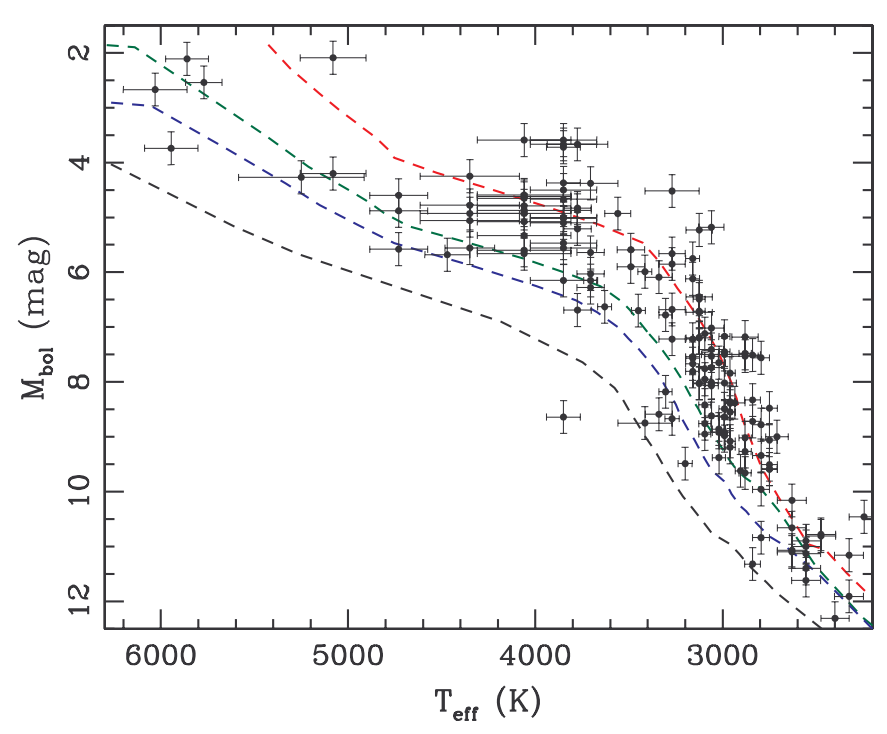

Figure A1. HR diagram for all members of our single star sample. The dashed lines denote isochrones at $1 \mathrm{Myr}$ (red), $5 \mathrm{Myr}$ (green), $10 \mathrm{Myr}$ (blue), and $50 \mathrm{Myr}$ (black). Most Taurus members fall along the 1-2 Myr isochrone, but many fall significantly below that level, perhaps due to the presence of an edge-on disk, undiscovered binary companion, erroneous observations. The highest-mass stars $\left(\gtrsim 1 M_{\odot}\right.$ ) also fall systematically below the $1-2 \mathrm{Myr}$ isochrone, suggesting either that the models might not be calibrated correctly in this regime or that these stars formed earlier in Taurus.

(A color version of this figure is available in the online journal.)

to Taurus $(\sim 170 \mathrm{pc}$ versus $\sim 145 \mathrm{pc})$. Otherwise, there is no significant trend in the ages of Taurus members, suggesting that global star formation proceeded nearly simultaneously (to within $\lesssim 0.3 \mathrm{Myr}$ ).

\section{APPENDIX B}

\section{THE COEVALITY OF TRIPLE AND QUADRUPLE SYSTEMS}

High-order multiple systems are a critical tool for constraining stellar evolutionary models. If these multiple systems form coevally, then they provide a simultaneous test of the models at three or more masses. This feature was exploited by White et al. (1999) to constrain models with the well-known quadruple system GG Tau and to infer the best set of models to use for low-mass stars (the Lyon models) as well as to establish the best temperature scale for young stars (Luhman et al. 2003). We now

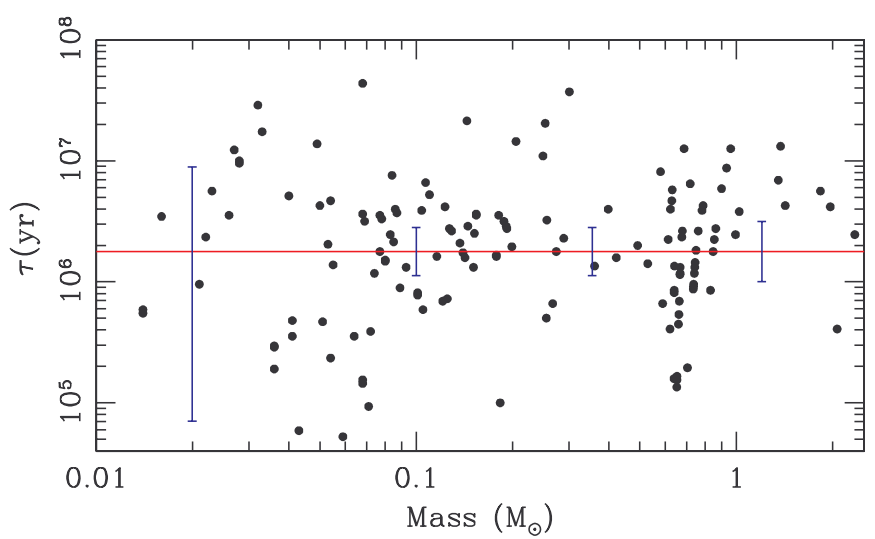

Figure A2. Age as a function of mass for all members of our single-star sample. We also show the model-derived median age of Taurus (1.8 Myr; red line) as determined from our sample, plus representative error bars at four different masses (blue). The mass-dependent age of our sample tracks the overall median age except at the high-mass end $\left(\gtrsim 1 M_{\odot}\right)$, where stars appear older, and at the low-mass end $\left(\lesssim 0.07 M_{\odot}\right)$, where the uncertainties become very large.

(A color version of this figure is available in the online journal.)

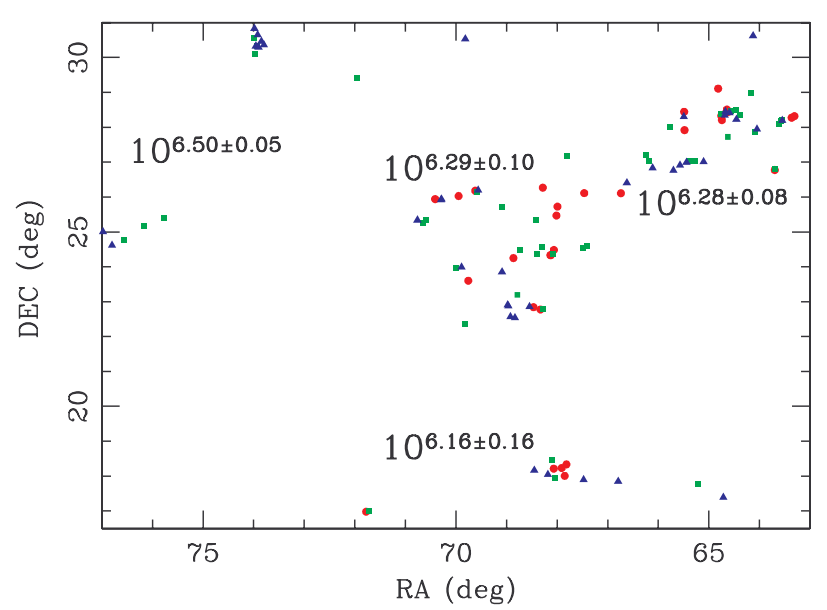

Figure A3. Spatial distribution for all members of our single-star sample in the mass range that is well calibrated $\left(0.07-0.9 M_{\odot}\right)$, color coded by age (red circles: <1 Myr; green squares: 1-3 Myr; blue triangles: $>3 \mathrm{Myr}$ ). We also show the mean age for the eastern subgroup, the southern subgroup, and the eastern and western halves of the core regions. The eastern subgroup appears $\sim 4 \sigma$ older than the other subgroups (3.2 Myr, vs. 1.4-1.9 Myr) and contains no stars with an apparent age of $<1 \mathrm{Myr}$, but otherwise there is no apparent pattern in the ages of Taurus members.

(A color version of this figure is available in the online journal.) 

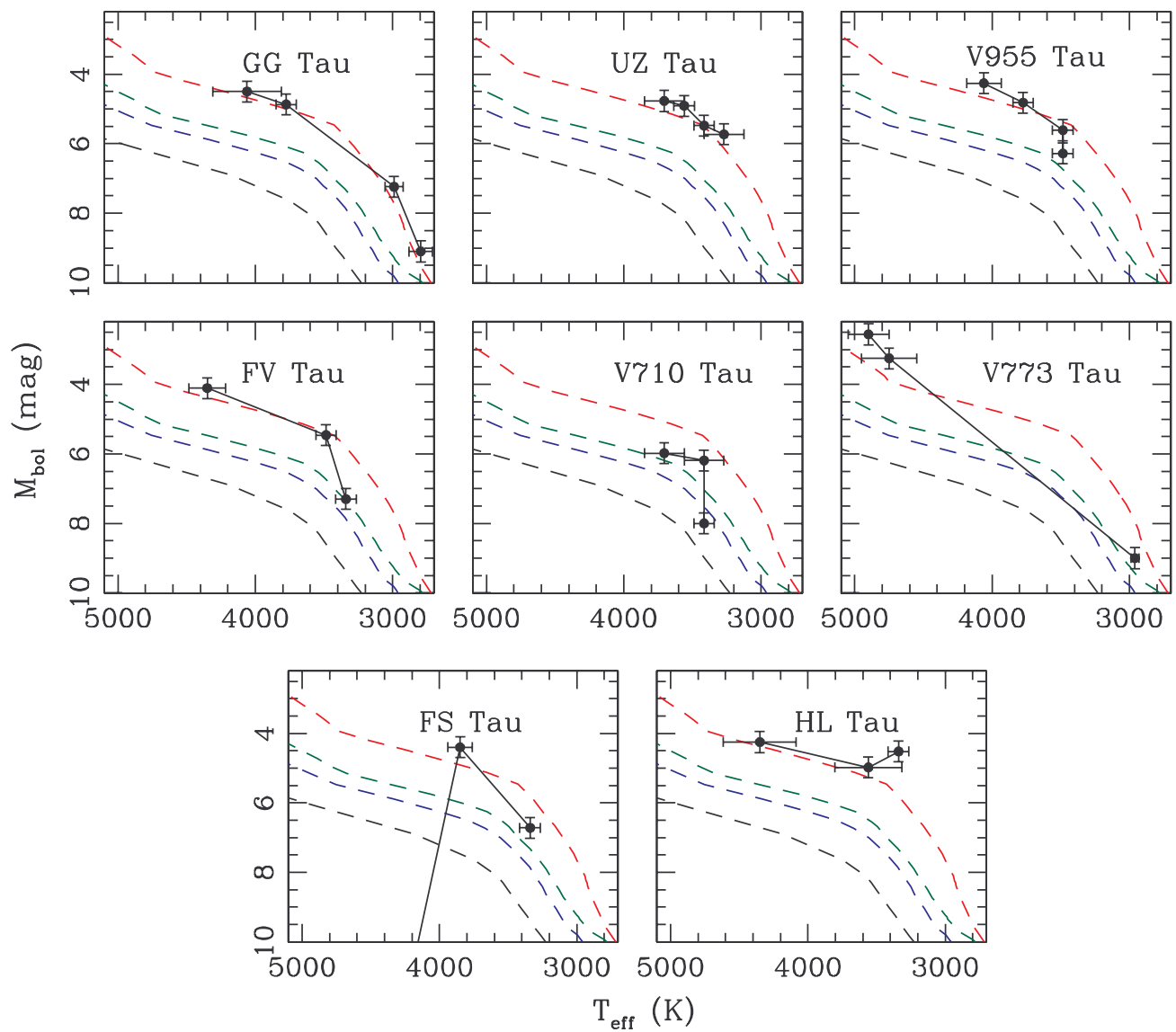

Figure B1. HR diagrams showing the components of eight hierarchical multiple systems. The four components of GG Tau and UZ Tau appear to be coeval, plus the components of V955 Tau might be coeval. However, as described in the text, the other five systems all have one or more components that disagree significantly. This could be due to errors in determining their luminosity (stars seen in scattered light only or which host a circumstellar disk) or temperature (incorrect spectral types).

(A color version of this figure is available in the online journal.)

extend this analysis to a quadruple system (UZ Tau), a quadruple that is part of a sextuple system (V955 Tau + LkHa332/ G2), three components each of two systems that are not yet completely characterized (FV Tau and V773 Tau), and three triple systems (FS Tau, V710 Tau, and HL Tau/XZ Tau), plus we replicate the analysis of White et al. (1999) for GG Tau to provide context.

As we show in Figure B1, all three of the quadruple systems appear to have consistent ages. The consistency of GG Tau is partly a result of its previous role in calibrating stellar models and temperature scales, but UZ Tau appears to be almost coeval and similarly consistent. Three components of V955 Tau + LkHa332/G2 also fall along the $1 \mathrm{Myr}$ isochrone, but V955 Tau B has an inferred age of $\sim 3 \mathrm{Myr}$. This is $\sim 2 \sigma$ away from a consistent age, but among 12 components, we would expect $\sim 0.6$ outliers at $\gtrsim 2 \sigma$.

Dynamical masses are available in the literature for the UZ Tau Aab and GG Tau Aab pairs and can be compared with those inferred from the HR diagram. The consistency is mixed. Guilloteau et al. (1999) found from the circumbinary disk kinematics that the total system mass for GG Tau Aab is $1.28 \pm 0.07 M_{\odot}$; the total mass predicted by theoretical models $\left(1.37 M_{\odot}\right)$ agrees to within $\sim 7 \%$. By contrast, Prato et al. (2002) reported dynamical masses for UZ Tau Aa and $\mathrm{Ab}$ of $1.02 \pm$ $0.06 M_{\odot}$ and $0.29 \pm 0.03 M_{\odot}$, while the masses predicted by theoretical models are 0.61 and $0.30 M_{\odot}$. The secondary mass agrees very well, but the discrepancy in the primary mass is very puzzling because its position in the HR diagram is virtually identical to that of GG Tau Ab, which has excellent consistency between observations and theory. Prato et al. explored the possible sources of this discrepancy in much greater detail, so we simply note its existence as a proof that HR diagram analysis plays a critical, but incomplete role in constraining stellar evolutionary models. A full study of evolutionary models must also include their dynamical masses (e.g., Schaefer et al. 2008), not just their temperatures and luminosities. Truly precise tests will also require direct measurement of radii (Stassun et al. 2008) rather than indirect estimates from the Stefan-Boltzmann law and the observed luminosity and temperature.

None of the three-component tests in our sample provides the same consistency seen among the quadruple systems, though as described in Section 3, the two largest discrepancies are likely to be systematic. The edge-on disk host Haro 6-5B sits very far below the Taurus sequence, unlike FS Tau AB, while V710 Tau $\mathrm{C}$ might also be seen in scattered light. HL Tau is also seen in scattered light in the optical, but our inferred age based on its $J$ magnitude seems consistent with that of XZ Tau A, suggesting that the central star of HL Tau might dominate its luminosity in the NIR. As described in Section 2, XZ Tau B was suggested to be a possible binary pair by Carrasco-Gonzalez et al. (2009), which would explain its apparent overluminosity. FS Tau A and FS Tau B also appear moderately discrepant, sitting $1.5 \sigma$ on either side of the $1 \mathrm{Myr}$ isochrone. FV Tau Aa and FV Tau Ba have very consistent ages, but FV Tau Bb sits somewhat lower in the HR diagram; the only spatially resolved spectrum for FV $\mathrm{Tau} \mathrm{Bb}$ is very noisy, so the apparent underluminosity could 
actually indicate that it has a later spectral type (M5.0-M5.5 rather than M3.5).

\section{REFERENCES}

Andrews, S., \& Williams, J. 2005, ApJ, 631, 1134

Ballesteros-Paredes, J., Hartmann, L., \& Vázquez-Semadeni, E. 1999, ApJ, 527, 285

Baraffe, I., Chabrier, G., Allard, F., \& Hauschildt, P. H. 1998, A\&A, 337, 403

Boden, A., et al. 2005, ApJ, 635, 442

Boden, A., et al. 2007, ApJ, 670, 1214

Briceño, C., Calvet, N., Kenyon, S., \& Hartmann, L. 1999, AJ, 118, 1354

Briceño, C., Hartmann, L., Stauffer, J., \& Martín, E. L. 1998, AJ, 115, 2074

Briceño, C., Luhman, K. L., Hartmann, L., Stauffer, J. R., \& Kirkpatrick, J. D. 2002, ApJ, 580, 317

Carpenter, J., Hillenbrand, L., \& Skrutskie, M. 2001, AJ, 121, 3160

Carpenter, J., Hillenbrand, L., Skrutskie, M., \& Meyer, M. 2002, AJ, 124, 1001

Carpenter, J., Mamajek, E., Hillenbrand, L., \& Meyer, M. 2006, ApJ, 651, 49

Carrasco-Gonzalez, C., Rodriguez, L., Anglada, G., \& Curiel, S. 2009, ApJ, 693, L86

Chabrier, G., Baraffe, I., Allard, F., \& Hauschildt, P. 2000, ApJ, 542, 464

Chabrier, G., Gallardo, J., \& Baraffe, I. 2007, A\&A, 472, 17

Cohen, M., \& Kuhi, L. 1979, ApJS, 41, 743

Correia, S., Zinnecker, H., Ratzka, Th., \& Sterzik, M. 2006, A\&A, 459, 909

D’Antona, F., \& Mazzitelli, I. 1997, Memorie della Societa Astronomia Italiana, 68,807

Delgado-Donate, E., Clarke, C., Bate, M., \& Hodgkin, S. 2004, MNRAS, 351, 617

Duchêne, G., Beust, H., Adjali, F., Konopacky, Q., \& Ghez, A. 2006, A\&A, 457,9

Duchêne, G., Ghez, A., McCabe, C., \& Weinberger, A. 2003, ApJ, 592, 288

Duchêne, G., Monin, J.-L., Bouvier, J., \& Menard, F. 1999, A\&A, 351, 954

Duquennoy, A., \& Mayor, M. 1991, A\&A, 248, 485

Elmegreen, B. 2000, ApJ, 530, 277

Fischer, D., \& Marcy, G. 1992, ApJ, 396, 178

Folha, D., \& Emerson, J. 1999, A\&A, 352, 517

Furlan, E., et al. 2006, ApJS, 165, 568

Ghez, A., Neugebauer, G., \& Matthews, K. 1993, AJ, 106, 2005

Gomez, M., Hartmann, L., Kenyon, S., \& Hewett, R. 1993, AJ, 105, 1927

Guieu, S., et al. 2006, A\&A, 446, 485

Guilloteau, S., Dutrey, A., \& Simon, M. 1999, A\&A, 348, 570

Haro, G., Iriarte, B., \& Chavira, E. 1953, Bol. Obs. Tonantzintla, 1, 3

Hartigan, P., Edwards, S., \& Ghandour, L. 1995, ApJ, 452, 736

Hartigan, P., \& Kenyon, S. 2003, ApJ, 583, 334

Hartigan, P., Strom, K., \& Strom, S. 1994, ApJ, 427, 961

Hartmann, L., et al. 2005, ApJ, 629, 881

Herbig, G., \& Bell, K. 1988, Lick Obs. Bull., 1111, 1

Herczeg, G., \& Hillenbrand, L. 2008, ApJ, 681, 594

Hillenbrand, L. 2009, in IAU Symp. 258, The Ages of Stars, ed. E. Mamajek, et al. (Cambridge: Cambridge Univ. Press), 81

Hillenbrand, L., \& White, R. 2004, ApJ, 604, 741

Indebetouw, R., et al. 2005, ApJ, 619, 931

Irwin, J., et al. 2007, MNRAS, 380, 541

Jensen, E., Mathieu, R., Donar, A., \& Dullighan, A. 2004, ApJ, 600, 789

Kenyon, S., \& Hartmann, L. 1995, ApJS, 101, 117

Konopacky, Q., Ghez, A., Rice, E., \& Duchêne, G. 2007, ApJ, 663, 394

Kraus, A., \& Hillenbrand, A. 2007a, ApJ, 662, 413

Kraus, A., \& Hillenbrand, A. 2007b, ApJ, 664, 1167

Kraus, A., \& Hillenbrand, L. 2008, ApJ, 686, L111

Kraus, A., \& Hillenbrand, L. 2009, ApJ, 703, 1511

Kraus, A., Ireland, M., Martinache, F., \& Lloyd, J. 2008, ApJ, 679, 762

Kraus, A., White, R., \& Hillenbrand, L. 2006, ApJ, 649, 306

Krist, J., et al. 1995, BAAS, 187, 4413

Krist, J., et al. 1998, ApJ, 501, 841

Luhman, K. 2000, ApJ, 544, 1044

Leggett, S. 1992, ApJS, 82, 351
Leggett, S., Allard, F., Berriman, G., Dahn, C., \& Hauschildt, P. 1996, ApJS, 104,117

Leinert, Ch., et al. 1993, A\&A, 278, 129

Lestrade, J.-F., et al. 1999, A\&A, 344, 1014

Liu, M., Najita, J., \& Tokunaga, A. 2003, AJ, 585, 372

Loinard, L., Torres, R., Mioduszewski, A., \& Rodriguez, L. 2008, Rev. Mex. AA, 34,14

Loinard, L., et al. 2007, ApJ, 671, 546

Lombardi, M., Lada, C., \& Alves, J. 2008, A\&A, 489, 143

Luhman, K. 2004, ApJ, 617, 1216

Luhman, K. 2006, ApJ, 645, 676

Luhman, K., Liebert, J., \& Rieke, G. 1997, ApJ, 489, L165

Luhman, K., Stauffer, J., Muench, Al, Rieke, G., Lada, E., Bouvier, J., \& Lada, C. 2003, ApJ, 593, 1093

Luhman, K., Whitney, B., Meade, M., Babler, B., Indebetouw, R., Bracker, S., \& Churchwell, E. 2006, ApJ, 647, 1180

Lyo, A.-R., Lawson, W., \& Bessell, M. 2004, MNRAS, 355, 363

Lyo, A.-R., Lawson, W., \& Bessell, M. 2008, MNRAS, 389, 1461

Mamajek, E., \& Hillenbrand, L. 2008, ApJ, 687, 1264

Martín, E., Dougados, C., Magnier, E., Menard, F., Magazzu, A., Cuillandre, J., \& Delfosse, X. 2001, ApJ, 561, 195

Masana, E., Jordi, C., \& Ribas, I. 2006, A\&A, 540, 735

Massarotti, A., Latham, D., Torres, G., Brown, R., \& Oppenheimer, B. 2005, AJ, 129,2294

Mathieu, R., Stassun, K., Basri, G., Jensen, E., Johns-Krull, C., Valenti, J., \& Hartmann, L. 1997, AJ, 113, 1841

McCabe, C., Ghez, A., Prato, L., Duchêne, G., Fischer, R., \& Telesco, C. 2006, ApJ, 636, 932

Meyer, M., Calvet, N., \& Hillenbrand, L. 1997, ApJ, 114, 288

Monin, J.-L., Clarke, C., Prato, L., \& McCabe, C. 2007, in Protostars and Planets V, ed. B. Reipurth, D. Jewitt, \& K. Keil (Tucson, AZ: Univ. Arizona Press), 395

Mouschovias, T. 1976, ApJ, 206, 753

Perrin, G., Coude Du Foresto, V., Ridgway, S., Mariotti, J.-M., Traub, W., Carleton, N., \& Lacasse, M. 1998, A\&A, 331, 619

Prato, L., Simon, M., Mazeh, T., Zucker, S., \& McLean, I. 2002, ApJ, 579, 99

Richichi, A., Ragland, S., \& Fabbroni, L. 1998, A\&A, 330, 578

Román-Zúñiga, C., Lada, C., Muench, A., \& Alves, J. 2007, ApJ, 664, 357

Sartoretti, P., Brown, R., Lathan, D., \& Torres, G. 1998, A\&A, 334, 592

Schaefer, G., Simon, M., Prato, L., \& Barman, T. 2008, AJ, 135, 1659

Schlegel, D., Finkbeiner, D., \& Davis, M. 1998, ApJ, 500, 525

Schmidt-Kaler, Th. 1982, Physical Parameters of the Stars, Landolt-Bornstein Numerical Data and Functional Relationships in Science and Technology, New Series, Group VI, Vol. 2b (Berlin: Springer)

Shu, F. 1977, ApJ, 214, 488

Shu, F., Adams, F., \& Lizano, S. 1987, ARA\&A, 25, 23

Simon, M., et al. 1995, ApJ, 443, 625

Skrutskie, M., et al. 2006, AJ, 131, 1163

Slesnick, C., Carpenter, J., Hillenbrand, L., \& Mamajek, E. 2006, AJ, 132, 2665

Stapelfeldt, K., Krist, J., Menard, F., Bouvier, J., Padgett, D., \& Burrows, C. 1998, ApJ, 502, 65

Stassun, K., Mathieu, R., Cargile, P., Aarnio, A., Stempels, E., \& Geller, A. 2008, Nature, 453, 1079

Stassun, K., Mathieu, R., \& Valenti, J. 2007, ApJ, 664, 1154

Steffen, A., et al. 2001, AJ, 122, 997

Stempels, H., Hebb, L., Stassun, K., Holtzman, J., Dunstone, N., Glowienka, L., \& Frandsen, S. 2008, A\&A, 481, 747

Strom, K., \& Strom, S. 1994, ApJ, 424, 237

Tanner, A., et al. 2007, PASP, 119, 747

Torres, R., Loinard, L., Mioduszewski, A., \& Rodriguez, L. 2007, ApJ, 671, 1813

van Belle, G., et al. 1999, AJ, 117, 521

Walter, F., Brown, A., Mathieu, R., Myers, P., \& Vrba, F. 1988, AJ, 96, 297

Weingartner, J., \& Draine, B. 2001, ApJ, 548, 296

White, R., \& Basri, G. 2003, ApJ, 582, 1109

White, R., \& Ghez, A. 2001, ApJ, 556, 265

White, R., Ghez, A., Reid, I. N., \& Schultz, G. 1999, ApJ, 520, 811

White, R., \& Hillenbrand, L. 2004, ApJ, 616, 998 